\title{
Mycopharmaceuticals and Nutraceuticals: Promising Agents to Improve Human Well-Being and Life Quality
}

\author{
Jameel R. Al-Obaidi $1, * \mathbb{C}$, Nuzul Noorahya Jambari ${ }^{2,3, *(\mathbb{D})}$ and E. I. Ahmad-Kamil ${ }^{4}$ \\ 1 Department of Biology, Faculty of Science and Mathematics, Universiti Pendidikan Sultan Idris, \\ Tanjong Malim 35900, Perak, Malaysia \\ 2 Department of Food Science, Faculty of Food Science and Technology, Universiti Putra Malaysia, \\ Serdang 43400, Selangor, Malaysia \\ 3 Laboratory of Food Safety and Food Integrity, Institute of Tropical Agriculture and Food Security, \\ Universti Putra Malaysia, Serdang 43400, Selangor, Malaysia \\ 4 Malaysian Nature Society (MNS), JKR 641, Jalan Kelantan, Bukit Persekutuan, \\ Kuala Lumpur 50480, Malaysia; ee_programs@mns.org.my \\ * Correspondence: jr_alobaidi@yahoo.com (J.R.A.-O.); noorahya@upm.edu.my (N.N.J.)
}

Citation: Al-Obaidi, J.R.; Jambari,

N.N.; Ahmad-Kamil, E.I.

Mycopharmaceuticals and

Nutraceuticals: Promising Agents to Improve Human Well-Being and Life Quality. J. Fungi 2021, 7, 503. https:// doi.org/10.3390/jof7070503

Academic Editors: Monika Gąsecka and Zuzanna Magdziak

Received: 2 June 2021

Accepted: 18 June 2021

Published: 24 June 2021

Publisher's Note: MDPI stays neutral with regard to jurisdictional claims in published maps and institutional affiliations.

Copyright: (c) 2021 by the authors. Licensee MDPI, Basel, Switzerland. This article is an open access article distributed under the terms and conditions of the Creative Commons Attribution (CC BY) license (https:/ / creativecommons.org/licenses/by/ $4.0 /)$.

\begin{abstract}
Fungi, especially edible mushrooms, are considered as high-quality food with nutritive and functional values. They are of considerable interest and have been used in the synthesis of nutraceutical supplements due to their medicinal properties and economic significance. Specific fungal groups, including predominantly filamentous endophytic fungi from Ascomycete phylum and several Basidiomycetes, produce secondary metabolites (SMs) with bioactive properties that are involved in the antimicrobial and antioxidant activities. These beneficial fungi, while high in protein and important fat contents, are also a great source of several minerals and vitamins, in particular $B$ vitamins that play important roles in carbohydrate and fat metabolism and the maintenance of the nervous system. This review article will summarize and discuss the abilities of fungi to produce antioxidant, anticancer, antiobesity, and antidiabetic molecules while also reviewing the evidence from the last decade on the importance of research in fungi related products with direct and indirect impact on human health.
\end{abstract}

Keywords: fungi; medicinal mushroom; myco-derived compounds; drug discovery

\section{Introduction}

Secondary metabolites (SMs) are essential players in fungal growth and development, and they are actively involved in the interactions with other organisms. Recently, interest in fungal SMs production and their function and mode of action drag high attention in drug discovery [1]. Most SMs are produced after the fungus has achieved its initial growth phase [2]. The fungal SM production process is influenced by the internal (genetics) [3] and external (environmental) factors [4], which includes the involvement of many successive enzymatic reactions essential for transforming primary metabolites sugars, lipids and amino acids into SMs during advanced stages of fungal growth [5], mainly during sporulation, virulence, intra- and interspecies signallings, defensive microbial mutualism, protection against abiotic stress, and reproductive development and form pigments [3]. Fungal SMs are either secreted into the environment or remain cell-attached by being incorporated into the structural elements within the cell [6,7]. Besides wild fungi, cultivated filamentous fungi in submerged flask-culture are also shown to have a high ability in producing functional SMs [8]. It is intriguing to understand how fungal SMs are involved in distinct functions, such as mediating intra- and interspecies communication, as well as regulating defence against competitors, nutrient acquisition, and symbiotic interactions [9].

Basidiomycetes, a major class of higher fungi, are capable of adjusting to different growth conditions, which result in the production of a variety of secondary metabolites. 
In sharp contrast to Ascomycota, Basidiomycota is highly diverse in its production, growth environment and morphology [10]. Most wild edible basidiomycetes propagate in the host plant roots. However, their rates of germination are low and only selected species are efficient at colonizing host plant roots via sporulation $[10,11]$. Although the exact number of basidiomycetes species is difficult to be estimated, it is agreed that about 14,000 mushrooms have been identified as basidiomycetes, in which about 7000 species are considered edible, and more than 2000 species are regarded as high-value edible mushrooms. Within these edible mushrooms, there are more than 700 species that are known to possess substantial pharmacological properties [12]. Edible mushrooms have an exceptional distinctive texture, taste, fragrance, and high nourishing value, and thus become highly valuable ingredients in epicure cuisine worldwide. Despite a widespread appreciation for edible mushrooms as a delicious alternative protein source, there are still a lot of concerns about consuming wild fungi $[13,14]$. Currently, there are more than a hundred mushroom species that can be cultivated $[15,16]$, yet less than thirty species are widely recognized as food and only a few are commercially produced [17]. Agaricus bisporus, Lentinus edodes, Pleurotus spp., and Flammulina velutipes are considered the most cultivated mushroom worldwide. While China is currently being the biggest mushroom producer, other mushroom-producing countries have also increased their production in the last decade [18].

Edible mushrooms are a rich source of distinctive SMs that are not found in other fungi, besides being natural product chemists as a source of hallucinogens such as Gymnopilus junonius mushroom [19], and pigments such as melanin from Auricularia auricula [20] and Termitomyces albuminosus [21]. Edible mushrooms are considered a food with high nutritive value and they have been used for a long time as functional food/nutraceuticals and medicinal remedies with economic significance [22,23]. They are considered an important source of essential nutrients as they are rich in protein and important fat contents [18], several minerals such as copper $(\mathrm{Cu})$, manganese $(\mathrm{Mn})$ and iron $(\mathrm{Fe})$ and vitamins such as vitamin B and C [24] that are involved in the metabolism of carbohydrates and fats (Figure 1). Even though wild mushroom price higher than cultivated mushrooms, there is still demand for consuming cultivated mushroom due to their constant availability [25]. For that reason, they could be considered an excellent source of many different nutraceuticals and could be used directly in a human diet to promote health [26,27]. With a large number of fungi have not been cultured and not well characterized, there is still significant work that needs to be conducted to grow uncultured fungi as a potential source of new chemicals with the potential for the discovery of new SMs with beneficial use for human [28]. Although fungi normally produce SMs in minute amounts due to the internal cellular regulatory mechanisms that regulate the low-level production, the amount produced is probably sufficient to increase the fungal growth competitiveness to other organisms and/or allow the fungi to coexist with other species in macrocosm [2,29].

In nature, fungi are confronted with multiple biotic and abiotic stressor that range from the competition and/or attack by other microorganisms, nutrient deficiency to changes in acidity, humidity, and temperature [30]. To maintain their sustenance and reproduction, fungi have developed several strategies for protection and communication, one of which is by producing various types of SMs. These fungal SMs increase the fungal protection against the invasion of predators, parasites, and diseases [31,32]. They may also be used to compete with other species and facilitate the reproductive processes [31,32]. In the last decade, the discovery of new fungal metabolites has accelerated tremendously [33]. However, with millions of fungal species to be identified in the future, there are many years of work to be conducted to increase the percentage of the discovery of these metabolites as an alternative source of natural pharmaceutical products [34]. Many compounds are produced by fungi with antioxidant, anticancer, antiallergic, antiobesity, immune-system-modulating, cardiovascular-protecting, anticholesterolemic, antimicrobial, detoxication, antitumor and inflammatory functions (Table 1) [35]. For a long time, edible fungi in general produce natural substances that usually have medicinal or nutraceutical activities as a promising source of new therapeutics. Those mushroom-related compounds such as $\beta$-glucans, 
other polysaccharide, vitamins and protein widely used in drug design and discovery with beneficial effects against dangerous diseases with less recorded side effects [36,37]. Polysaccharides considered one of the most important molecules for modern pharmaceutical research due to their flexibility to act as drug delivery agents especially for cancer therapy [38]. Many metabolites produced by edible fungi are known to be unique bioactive compounds that can be found in the fruiting bodies and liquid cultured mycelium $[39,40]$. Modern biotechnology research like omics-based research on fungi has revealed that many edible/medicinal species are beneficial for the inhibition and treatment of some enduring diseases, such as cancer, brain function cardiovascular diseases, diabetes and degenerative nerve diseases [41]. In light of the emerging literature, the objective of this chapter is to compile the more recent evidence about the importance of SMs which play important roles in fungal defence and/or signalling and with high potential health benefits to human.

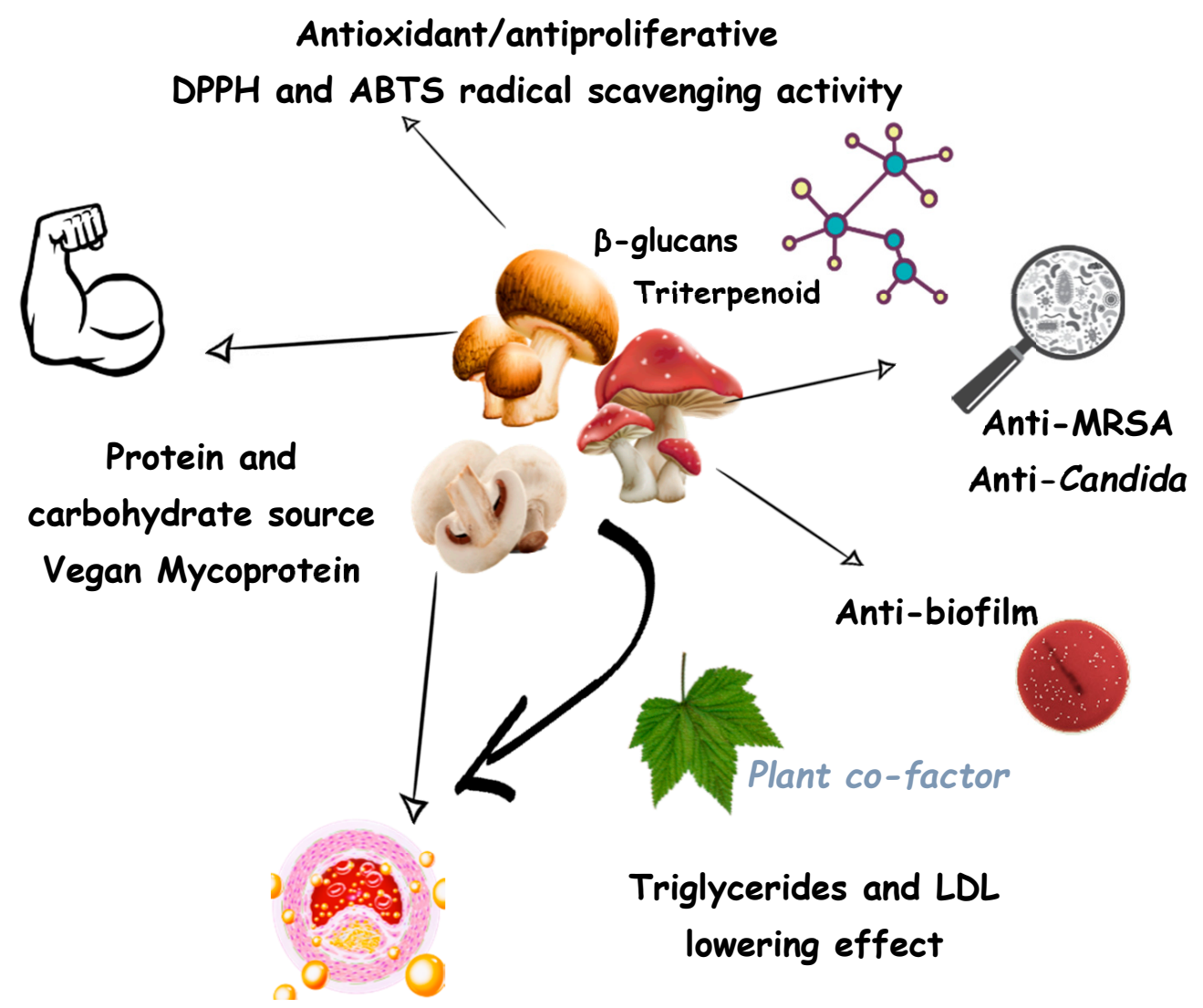

Figure 1. Schematics overview of edible mushroom contribution to human health.

Table 1. Examples of major compounds from fungi and their main potential benefits to human health reported between 2010 and 2020.

\begin{tabular}{cccc}
\hline Fungal Species & Active Molecules & Effect & Reference \\
\hline Colletotrichum capsici & Taxol & Anticancer (mitotic inhibitor) & [42] \\
\hline Ganoderma lucidum & C-19 fatty acids & against HL-60 & [43] \\
\hline $\begin{array}{c}\text { Penicillium buchwaldii and } \\
\text { Penicillium spathulatum }\end{array}$ & asperphenamate & Anticancer & [44] \\
\hline Schizophyllum commune & hydrophobin SC3 & Anticancer (sarcoma S180 cell line) & [45] \\
\hline Fusarium solani & Taxol, baccatin III & Anticancer (HeLa) & [46] \\
\hline
\end{tabular}


Table 1. Cont.

\begin{tabular}{|c|c|c|c|}
\hline Fungal Species & Active Molecules & Effect & Reference \\
\hline Flammulina velutipe & FIP-fve & Anticancer (A549) & {$[47]$} \\
\hline Gomphus clavatus & gCG-1 & $\begin{array}{l}\text { Antioxidant (against activity (apoptosis } \\
\text { of HepG-2)) }\end{array}$ & [48] \\
\hline Ganoderma atrum & FIP-gat & Antioxidant (against MDA-MB-231) & [49] \\
\hline Lignosus rhinocerotis & FIP-Lrh & Anticancer (HeLa, A549, MCF-7) & [50] \\
\hline Aspergillus candidus & 3-Hydroxyterphenyllin (3-HT) & $\begin{array}{l}\text { Anticancer (ovarian carcinoma cell } \\
\text { lines, A2780/) }\end{array}$ & [51] \\
\hline Ramaria botrytis & ubiquitin-like & $\begin{array}{c}\text { Anticancer (293T, HeLa A549, KB and } \\
\text { MCF-7) }\end{array}$ & [52] \\
\hline Fusarium solani & (FIP-nha) & A549 apoptosis & [53] \\
\hline Cerrena unicolor & ex-LMSI, ex-LMSII, and ex-LMSIII & $\begin{array}{l}\text { Anticancer (MDA-MB-231, PC3, and } \\
\text { MCF7) }\end{array}$ & {$[54]$} \\
\hline Trichoderma viride & $\begin{array}{l}\text { 3-beta-hydroxy urs-12-en-28-oic } \\
\text { acid }\end{array}$ & Anticancer (HeLa) & [55] \\
\hline Poria cocos & Triterpenes & Anti-Hyperglycemic & [56] \\
\hline Pleurotus tuber-regium & polysaccharides (1P, 2P, and 3P) & Anti-Hyperglycemia & [57] \\
\hline Aspergillus oryzae & $\mathrm{P}-1$ and $\mathrm{P} 2$ peptide & $\alpha$-Glucosidase Inhibitory & [58] \\
\hline $\begin{array}{c}\text { Agaricus blazei, Coprinus } \\
\text { comatus, Cordyceps militaris, } \\
\text { Inonotus obliquus, Phellinus } \\
\text { linteus }\end{array}$ & $\begin{array}{l}\text { p-coumaric acid, p-hydroxybenzoic } \\
\text { acid and cinnamic acid }\end{array}$ & Inhibition of $\alpha$-amylase & [59] \\
\hline Ganoderma lucidum & (WEGL) mycelium & reduces obesity & {$[60]$} \\
\hline Penicillium digitatum & AfpB protein & Antifungal activity & {$[61]$} \\
\hline $\begin{array}{l}\text { Pleurotus ostreatus and } \\
\text { Pleurotus florida }\end{array}$ & Methanolic extracts & Antimicrobial activity & {$[62]$} \\
\hline $\begin{array}{l}\text { Monascus purpureus, } \\
\text { Aspergillus oryzae, Neurospora } \\
\text { intermedia, Fusarium venenatum }\end{array}$ & Fungal biomass & Vegan protein & [63] \\
\hline Agaricus blazei & Multi-vitamins & $\begin{array}{c}\text { Immune sustem stimulators, } \\
\text { antimicrobial }\end{array}$ & {$[64]$} \\
\hline
\end{tabular}

\section{Fungi Contribution as Antioxidant and Anticancer Products}

Fungi including very popular, affordable and widely utilized mushrooms are a great source of a high-quality natural products with good potential anticancer and antioxidant agents that could be further studied and clinically tested for a future anticancer drug [65]. The white common mushrooms, A. bisporus, is currently the most widely cultivated and most studied edible mushroom worldwide. It is consumed due to its pleasant flavour and good natural source of vitamin B [66]. A study conducted on the digested protein from the common mushroom showed that it possessed natural functional properties in suppressing oxidative stress and suggested its potential application in the food industry as alternative natural antioxidants [67]. This white mushroom has been extensively studied and many reports have examined and discussed its antioxidant ability from both wild and cultivated Agaricus species from different parts of the world [67-71]. The debate regarding the vast fungal biodiversity linked to direct the resources for the discovery of new SMs especially from newly identified fungi species which needs further research to characterize those SMs and their mode of action. In this regard, research conducted on different Agaricus species revealed the number of phenolic compounds varied with dried Aspergillus brasiliensis extracts revealed the highest concentration content of 1 phenolic acid $(33.9 \mathrm{mg} / 100 \mathrm{~g})$ 
while A. bitorquis showed the highest scavenge 2,2-diphenyl-1-picrylhydrazyl (DPPH) radicals ability [72]. Previous studies showed that Agaricus blazei possessed potential anticancer and antiproliferation properties mainly due to its ability to produce betaglucans and polysaccharides [73], and hot extracts of these fungi were shown to have an apoptotic effect on human cancer cell lines $[74,75]$. The fungus proved on a clinical trial to be safe for long term consumption [76] with many commercial nutraceuticals products available in the market [77]. Despite the debate of the proper scientific name, the traditional fungus Sanghuangporus sanghuang growing on mulberry is believed to have medicinal value and used to treat inflammation [78]. Triterpenoid extracted from the mycelium of this fungus exhibits antioxidant activity against hydroxyl radicals, $\left(2,2^{\prime}\right.$-azinobis(3-ethylbenzothiazoline-6-sulfonic acid) (ABTS) and DPPH free radicals [79]. However, more in vitro studies on the antioxidant mechanism and active compounds are needed to support those findings. Storage temperature and fungal nutrition were also shown to have an impact on the production of high-quality antioxidant compounds. Low temperature appears to be a reduction factor, and lower temperature showed lower antioxidant activity in a range of +25 to $-40{ }^{\circ} \mathrm{C}$ [80] while the presence of essential minerals like zinc (Zn) and selenium (Se) increased the antioxidant related metabolites produced such as ascorbic acid [81]. The effect of medium composition on the antioxidant and anticancer activity of cultivated endophytic fungi was further investigated where Talaromyces purpureogenus isolated from seaweed showed the highest antioxidant activity when they were grown on potato dextrose agar, but anticancer activity against HeLa, MCF-7 and HePG2 cell lines respectively appear to be at the highest level when this species was cultivated in malt extract broth [82]. Exopolysaccharides from Fusarium oxysporum isolated from tropical Otoba gracilipes leaves and cultivated using Potato dextrose broth revealed higher antioxidant ability compared to the extract from the same fungi that were grown on potato dextrose-yeast extract broth (PDYB) [83]. A group of Fusarium species that are isolated from the fritillary bulb were shown to produce compounds such as rutin, phlorizin, 2,4-ditert-butylphenol and 2,6-di-tert-butyl hydroquinone with antioxidant activity as measured using DPPH and antioxidant ABTS, HPLC and gC-MS. Phenolic, flavonoid, and saponin compounds from the fungus Fritillaria unibracteata exhibited potential activity to remove reactive oxygen species with potential novel antioxidant compound [84]. Toledo and his collaborators work conducted using gas chromatography with flame ionization detection gC-FID and ultra-fast liquid chromatography coupled to a photodiode array detector (UFLC-PDA) have revealed antioxidant activities from nine different edible mushrooms in Argentina and Ramaria patagonica showed the highest antioxidant activities and the highest phenolic content represented by the presence of gallic, cinnamic acids, p-coumaric and, p-hydroxybenzoic [85]. Oyster mushroom (Pleurotus ostreatus) is considered one of the very important edible mushrooms with high medicinal and nutritional value [86]. Different stages of the mushroom were examined for antioxidant activity, and the DPPH and ABTS radical scavenging activity test showed the highest levels in polyphenols from the fruiting body [87]. Pleurotus tuber-regium contains polysaccharide with antitumor activity [88] and a study on the proteomic changes in this mushroom revealed increase rate of mycelium growth and polysaccharide production as an effect of adding Tween 80 [89]. A proteomic study on tiger milk mushroom identified pharmacological-related proteins besides other proteins involved in defence and metabolism. In that study, proteins like subtilin-like serine were found to have potential anticancer activity against breast cancer cells [90]. A recent review on the pharmaceutical abilities of the tiger milk mushroom discussed the anticancer, antimicrobial and antiasthmatic properties of tiger milk mushroom [91].To investigate the anticancer activities of polysaccharides isolated from edible mushrooms, a proteomic study was conducted on HepG2 cells upon the treatment with polysaccharides from Ganoderma lucidum, Auricularia auricular and Phellinus linteus, and found that changes detected in the lung cancer cell line proteins might lead to HepG2 apoptosis [92]. Colon cancer HT-29 cell death was induced by low molecular weight (LMW) extracts from the cultivated rot fungus Cerrena unicolor with no negative effect 
in the control of normal cell lines [93]. The same fungus in earlier research showed that LMW extracts have the same potential anticancer activity in breast (MDA-MB-231, MCF7) and prostatic (PC3) cancer cell lines besides antibacterial activity against some human pathogenic bacteria like Bacillus subtilis, Staphylococcus aureus, and Escherichia coli [54]. Extraction methods of phenolic compounds always play an important role in the quality of the antioxidant obtained from edible mushroom [94]. The 50\% ethanol proved to be the most efficient method when several dry edible mushrooms were compared for their phenolic compounds and antioxidant activities, and shitake mushroom (L.edodes) showed the highest [95]. While 70\% ethanol exhibited the highest antioxidant activity of termite mushroom (Termitomyces spp.), hot water extract proved to be the method of choice to get the highest scavenge ability when it related to wood ear mushroom (Auricularia spp.) [96]. A comparison between hot water extracts from edible mushroom antioxidant activities showed higher activity of antioxidants from Ganoderma lucidum compared to other fungi like Schizophyllum commune [97]. Low molecular fraction (ex-LMS), Laccase (ex-LAC) and endo-polysaccharides (c-EPL) isolated from the basidiomycete fungi Cerrena unicolor with the first (ex-LMS) showing the highest reduction capacity using DPPH assay. Besides that, the identified metabolites showed also antibacterial activity against Escherichia coli (ex-LAC, ex-LMS) and more effectively against Staphylococcus aureus (c-EPL, ex-LMS) [98]. Rhodiola spp. Alpine endophytic fungi examined by Cui and coresearchers when they identified more than 100 metabolites linked to more than 300 species. The study revealed phenolic and flavonoid compounds with antioxidant properties that reached up to $90 \%$ of DPPH radical-scavenging rates such as Rct45, Rsc57, and Rct63 from plant species Rhodiola crenulata with high potential for the production of these antioxidants by artificial fungal cultivation [99]. Lion's mane edible mushroom (Hericium erinaceus) is known for its bioactive compound with antibacterial, antitumor, and immune-modulating properties [100]. Proteomic study of the mycelium and fruiting body of this mushroom revealed proteins with potential function in carbohydrate metabolism, cell signaling, and sterol production and suggested potential pharmacological properties for many polysaccharides [101]. Boletus spp. is considered a wild edible mushroom rich with compounds that have antioxidant properties [102,103]. The antioxidant ability of the Boletus edulis (together with Xerocomus badius) cooked for consumption was tested and revealed a high concentration of phenols, flavonoid antioxidant activity and vitamin content [104]. Later, 13 different Boletus species were compared for antioxidant ability, Boletus luridus showed very high antioxidant ability with potential natural nutraceuticals product from this species [105]. Transcriptomic study on the edible mushroom L. edodes showed significant changes in expression between mycelium and the fruiting body with developmental stages specific protein identified and linked to potential antioxidant properties [106]. Tiger milk mushroom (Lignosus rhinocerus) is known for its valuable health properties, yet it is hard to be found in nature and many efforts have been made to cultivate it. Cultivated tuber exhibited antioxidant activities and anticancer properties against human breast cancer cell line (MCF7) and human lung cancer cell line (A549) with no toxic effect on normal human lung cell line (MRC5) [107]. A group of Ascomycota endophytic fungi have shown antioxidant potential were isolated from the stem of the mangrove species Rhizophora stylosa and Rhizophora mucronata with more than $80 \%$ of those antioxidant compounds showing antioxidant ability. Both HHL38 and HHL55 recorded the highest natural antioxidant capacity [98]. Not only endosymbiont fungi but some pathogenic filamentous species like Aspergillus can infect human (Figure 2) (e.g., Aspergillus fumigatus), animals (e.g., Aspergillus flavus), and plant (Aspergillus niger) and also can be used in food production (Aspergillus oryzae) [108]. A study on the growth of A. unguis on different media showed the ability of this fungus to produce metabolites with high antioxidant and antimicrobial activity when cultivated using potato dextrose agar [109]. Another filamentous fungus, Mucor circinelloides, was tested for antioxidant content by comparing different strains cultivated and extracted using different extraction methods, the ethanolic-based extraction method from the strain MC277.49 cultivated for 5 days showed the highest antioxidant content after testing with the $\beta$-carotene bleaching 
assay, ABTS scavenging activity. Certainly, there is an increasing need in discovering and producing antioxidant, anticancer therapeutic agents efficiently from natural sources that have low toxicities and less impact on the environment. Myconutrients product is one of the sources to meet these measures. Such products are secondary metabolites in nature has a varied range of applications in medicinal and drug discovery opportunities.

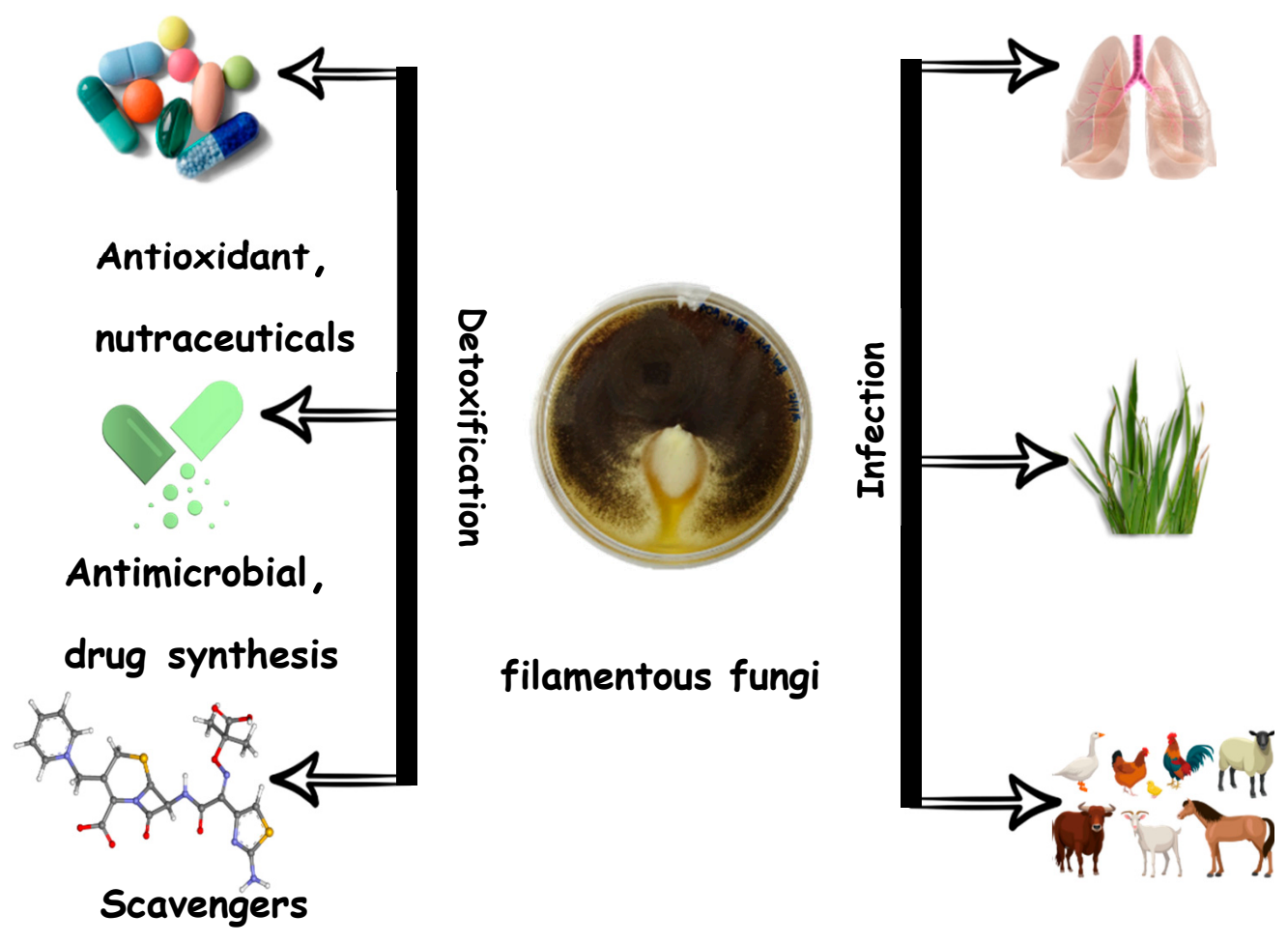

Figure 2. Pathogenic filamentous fungi potential and positive interactions with the human.

Due to the extensive structural variety, complexity and various pharmaceutical characteristic of identified metabolites, this area has been more interesting for researchers and more research on animal experiment and clinical trials are needed to verify the validity of those claims towards supplement/drug production at the industrial level.

\section{Fungi as Protein and Carbohydrate Source}

Generally, fungi can be considered as a source of cholesterol-free protein and carbohydrate and a good meat substitute that is produced via low carbon footprint processes, such as commercially cultivated Fusarium venenatum [110,111].

Fungal polysaccharides are known to possess great antioxidant activities, as have been shown in Lentinus edodes [112], Grifola forndosa [113] and Leucopaxillus giganteus [114]. The functional and nutritive properties of fungi as dietary protein sources have also been discussed and reviewed elsewhere [115]. While edible mushrooms are generally regarded as containing a high level of proteins and carbohydrates, further investigation on the studies performed for the last 10 years indicates that the fungal nutritive characteristics vary depending on the genera of the fungi, wild or cultivated, the geographical location, and the cultivation media (Table 2). Some of these edible wild fungi from different geographical origins have relatively high protein contents such as Hygrocybe parvula (36.5\%), Calocybe cornucopioides (47.2 g/100g), and Boletus edulis (39.0\%) while some have relatively poor protein content such as wild desert truffle, Terfezia boudieri Chatin. Cultivated fungi from different species seemed to fare better in their protein contents such as Volvariella volvacea (32\%), Pleurotus pulmonarius RN82 (43.07\%), Tricholoma, Shiitake mushroom (37.23\%), Pleurotus sajor-caju (36.75\%), and yeast, Candida valida (44.3\%), although the compost medium composition did affect the protein content greatly. Some specific species of edible fungi 
possess high carbohydrate contents such as Marcolepiota procera $(83.65 \mathrm{~g} / 100 \mathrm{~g})$, Boletus regius $(88.79 \mathrm{~g} / 100 \mathrm{~g})$, Lentinus torulosis $(68.24 \mathrm{~g} / 100 \mathrm{~g})$, Armillaria mellea $(71.28 \mathrm{~g} / 100 \mathrm{~g})$, Boletus aereus Bull. (72.83\%), L. deliciosus (76.0\%). Yet species such as Ganoderma lucidum (Leyss ex Fr.) Karst. Yashan was shown to have low protein and carbohydrate contents at $9.31 \%$ and $0.54 \%$ respectively. While these findings mostly measured crude protein and carbohydrate contents only, it would be interesting to investigate their specific amino acid and protein composition as well as polysaccharides as sources of functional food for future work.

Several wild mushrooms reveal their importance as sources of carbohydrate and essential minerals, for instance, the termite mushroom, Termitomyces heimii [116], Termitomyces microcarpus and Termitomyces tyleranus [24]. Much molecular-based research revealed the protein and carbohydrate biosynthesis pathways and how this could be utilized in applications of nutritious/functional food production from fungi. Transcriptomic and proteomic studies on $A$. aegerita revealed that the annotated genes and peptide steroid biosynthesis were upregulated in the mycelium whereas the polysaccharide biosynthesisrelated genes were upregulated in the fruiting bodies with higher associated peptides were produced [117]. Transcriptomic de novo assembly of the rare edible fungi, Leucocalocybe mongolica (S. Imai), using Illumina paired-end sequencing technology were able to identify genes potentially involved in, steroid, terpenoid, and unsaturated fatty acids biosynthesis and were annotated to play a vital role in the metabolism of nutrients [118]. This information could also be helpful for the cultivation of rare species [118]. Recently, the brown mycelium features of Shiitake mushroom (L.edodes) were investigated using comparative transcriptomics analysis. The study revealed the role of brown mycelium in cell wall synthesis, light sensing, reduction of oxygen, and metabolic of carbohydrates [119]. Different cultivation components were compared during the cultivation of the edible mushroom Grifola frondosa (Maitake) CE-MS-based metabolomics study revealed differences in amino acid and organic acid in the strain gF433 with high amount compared to the stain Mori52, which may reveal high efficiency of the stain gF433 with high metabolite content and product efficiency [120]. Research conducted using a pea byproduct as a substrate for fungi growth with $A$. oryzae showed the highest ability to produce protein mass (54\% of the total mass). The promising findings could be a start point to consider the production of vegan mycoproteins at the industrial scale [63]. The same species can produce protein mass from wastewater from starch plants which serve well as a good source of animal feed [121]. Enokitake mushroom has many health properties reported [122], a metabolomic study on this edible mushroom revealed 16 different potential biomarker metabolites involved in glutamate metabolism, tricarboxylic acid (TCA) cycle, carbohydrate metabolism, arginine and proline metabolisms, while also revealed the liver-protective effects of this mushroom metabolites in vivo on acute liver injury rats [123].

Table 2. List of studies on protein and carbohydrate contents in edible fungi from different geographical locations from 2010-2021.

\begin{tabular}{|c|c|c|c|c|}
\hline $\begin{array}{l}\text { Fungal } \\
\text { Species }\end{array}$ & Wild or Cultivated & Location & Composition & References \\
\hline $\begin{array}{l}24 \text { Chilean wild and commercial } \\
\text { edible mushrooms from genera } \\
\text { Agaricus, Agrocybe, Boletus, } \\
\text { Cortinarius, Cyttaria, Flammulina, } \\
\text { Grifola, Lactarius, Lentinus, } \\
\text { Macrolepiota, Morchella, Pleurotus, } \\
\text { Ramaria, Suillus, Tricholoma, and } \\
\text { Xeroco-mus }\end{array}$ & $\begin{array}{l}\text { Wild and cultivated } \\
\text { mushrooms }\end{array}$ & $\begin{array}{l}\text { Nuble and Bio-Bio } \\
\text { Regions, Chile }\end{array}$ & $\begin{array}{l}\text { Crude protein content: } \\
8.56-23.88 \text { g/100 g d.w. } \\
\text { (Highest in Cortinarius lebre } \\
\text { (Chilean endemic } \\
\text { mushroom)); Carbohydrate } \\
\text { content: } \\
62.97-83.65 \text { g/100 g d.w. } \\
\text { (highest in Marcolepiota } \\
\text { procera); }\end{array}$ & [124] \\
\hline
\end{tabular}


Table 2. Cont.

\begin{tabular}{|c|c|c|c|c|}
\hline $\begin{array}{l}\text { Fungal } \\
\text { Species }\end{array}$ & Wild or Cultivated & Location & Composition & References \\
\hline Volvariella volvacea & Cultivated mushroom & Solan, India & $\begin{array}{l}\text { Protein content: } 32 \% \\
\text { Carbohydrate content: } \\
52.2 \%\end{array}$ & [125] \\
\hline $\begin{array}{l}\text { Clavaria rosea, Ganoderma sp., } \\
\text { Geastrum triplex, Hygrocybe } \\
\text { parvula, Schleroderma bermudense }\end{array}$ & Wild mushrooms & $\begin{array}{l}\text { Shivamogga } \\
\text { District, Karnataka, } \\
\text { India }\end{array}$ & $\begin{array}{l}\text { Protein contents: } \\
25.71-36.51 \% \text { (highest in } \\
\text { Hygrocybe parvula); } \\
\text { Carbohydrate } \\
\text { contents:37.38-48.63\% } \\
\text { (highest in Ganoderma sp.) }\end{array}$ & [126] \\
\hline $\begin{array}{l}\text { Pleurotus pulmonarius RN2, P. } \\
\text { djamor RN81 and RN82 }\end{array}$ & $\begin{array}{l}\text { Cultivated mushrooms } \\
\text { (cultivated on rice } \\
\text { straw (Oryza sativa L.), } \\
\text { corn stubble and husk } \\
\text { (Zea maize L.)) }\end{array}$ & USA and Panama & $\begin{array}{l}\text { Protein contents: } \\
23.54-43.07 \% \text { (highest in } \\
\text { RN82 cultivated on corn } \\
\text { husk); carbohydrate } \\
\text { contents: } 27.39-52.44 \% \\
\text { (highest in RN2 cultivated } \\
\text { on corn stubbles) }\end{array}$ & [127] \\
\hline $\begin{array}{l}\text { Lentinus sajor-caju and Lentinus } \\
\text { torulosus }\end{array}$ & Wild mushrooms & $\begin{array}{l}\text { Similipal Biosphere } \\
\text { Reserve, India }\end{array}$ & $\begin{array}{l}\text { protein content: } 27.31-28 . \\
36 \mathrm{~g} / 100 \mathrm{~g} \text {; carbohydrate } \\
\text { content: } 64.95-68 . \\
24 \mathrm{~g} / 100 \mathrm{~g} .\end{array}$ & [128] \\
\hline $\begin{array}{l}\text { Amanita crocea (Quél. in Bourd.) } \\
\text { Singer ex Singer, Amanita mairei } \\
\text { (Foley), Boletus porosporus (Imler } \\
\text { ex Bon E g. Moreno), Boletus } \\
\text { regius (Krombh.), Gyromitra } \\
\text { esculenta (Pers. ex Pers.) Fr., } \\
\text { Helvella lacunose (Afzel.), Russula } \\
\text { aurea Pers., Russula virescens } \\
\text { (Schaeff.) Fr. }\end{array}$ & Wild mushrooms & $\begin{array}{l}\text { Bragança } \\
\text { (Northeast } \\
\text { Portugal) }\end{array}$ & $\begin{array}{l}\text { Protein content: } \\
4.40-21.85 \mathrm{~g} / 100 \mathrm{~g} \text { d.w. } \\
\text { (highest in Rusula } \\
\text { virenscens); Carbohydrate } \\
\text { content: } \\
49.64-88.79 \mathrm{~g} / 100 \mathrm{~g} \text { d.w. } \\
\text { (highest in Boletus regius). }\end{array}$ & [129] \\
\hline Agaricus bohusii Bon & Wild mushroom & $\begin{array}{l}\text { Jabučki rid, } \\
\text { Northern Serbia }\end{array}$ & $\begin{array}{l}\text { Protein content: } \\
18.06 \text { g/100 g dw; } \\
\text { carbohydrate content: } \\
69.79 \text { g/100 g d.w. }\end{array}$ & [130] \\
\hline $\begin{array}{l}\text { Fistulina hepatica, } \\
\text { Infundibulicybe geotropa, } \\
\text { Laetiporus sulphureus, } \\
\text { Macrolepiota procera var. procera } \\
\text { and Suillus granulatus }\end{array}$ & Wild mushrooms & $\begin{array}{l}\text { Sicily, Southern } \\
\text { Italy }\end{array}$ & $\begin{array}{l}\text { Protein contents: } \\
1.31-4.37 \mathrm{~g} \% \text { (highest in } L . \\
\text { sulphureus); carbohydrate } \\
\text { contents: } 2.08-4.57 \mathrm{~g} \% \\
\text { (highest in } I . \text { geotropa) }\end{array}$ & [131] \\
\hline $\begin{array}{l}\text { Cantharellus isabellinus, C. cibarius } \\
\text { var. longipes, C. rhodophyllus, } \\
\text { C. miniatescens, C. appalachiensis, } \\
\text { C. cibarius, C. natarajanii, } \\
\text { C. fibrillosus, C. lateritius, } \\
\text { C. applanatus, Cr. cibarius var. } \\
\text { intermedius C. himalayensis, } \\
\text { C. elongatipes, C. cibarius var. } \\
\text { multiramis, C. indicus, } \\
\text { C. pseudoformosus, C. umbonatus, } \\
\text { C. minor }\end{array}$ & Wild mushrooms & $\begin{array}{l}\text { Northwestern } \\
\text { Himalayas, India }\end{array}$ & $\begin{array}{l}\text { Protein: } 21.6-43.2 \mathrm{mg} / \mathrm{g} \\
\text { (highest in C. miniatescens); } \\
\text { carbohydrate: } \\
9.94-26.5 \mathrm{mg} / \mathrm{g} \text { (highest in } \\
\text { C. minor) }\end{array}$ & [132] \\
\hline
\end{tabular}


Table 2. Cont.

\begin{tabular}{|c|c|c|c|c|}
\hline $\begin{array}{l}\text { Fungal } \\
\text { Species }\end{array}$ & Wild or Cultivated & Location & Composition & References \\
\hline $\begin{array}{l}\text { Armillaria mellea (Vahl) P. Kumm., } \\
\text { Calocybe gambosa (Fr.) Donk, } \\
\text { Clitocybe odora (Fr.) P. Kumm., } \\
\text { Coprinus comatus (O.F. Müll.) } \\
\text { Pers. }\end{array}$ & Wild mushrooms & $\begin{array}{l}\text { Bragança, } \\
\text { Northeast Portugal }\end{array}$ & $\begin{array}{l}\text { Protein: } \\
\text { 15.46-17.33 g/100 g dw } \\
\text { (highest in Clitocybe odora); } \\
\text { carbohydrates: } \\
69.83-71.28 \mathrm{~g} / 100 \mathrm{~g} \mathrm{dw} \\
\text { (highest in Armillaria } \\
\text { mellea) }\end{array}$ & [133] \\
\hline $\begin{array}{l}\text { Pleurotus florida, P. sajor-caju and } \\
\text { P. ostreatus }\end{array}$ & $\begin{array}{l}\text { Cultivated mushrooms } \\
\text { (cultivated on bean } \\
\text { straw) }\end{array}$ & Pantnagar, India & $\begin{array}{l}\text { Protein contents: } \\
30.92-36.75 \% \text { db (highest in } \\
\text { Pleurotus sajor-caju); } \\
\text { carbohydrate contents: } \\
0.49-31.59 \% \text { db (highest in } \\
\text { Pleurotus florida) }\end{array}$ & [134] \\
\hline $\begin{array}{l}\text { Agaricus campestris, Boletus edulis, } \\
\text { Calocybe gambosa, } \\
\text { Cantharelluscibarius, Calocybe } \\
\text { cornucopioides, Entoloma } \\
\text { clypeatum, Flammulina velutipes, } \\
\text { Macroleptiotaprocera, M. elata, } \\
\text { Pleurotus ostreatus }\end{array}$ & Wild mushrooms & $\begin{array}{l}\text { Croatian regions of } \\
\text { Istria (northwest) } \\
\text { and Slavonia } \\
\text { (northeast) }\end{array}$ & $\begin{array}{l}\text { Protein: } \\
24.22-47.21 \mathrm{~g} / 100 \mathrm{~g} \mathrm{dw} \\
\text { (highest in } \\
\text { C. cornucopioides); } \\
\text { carbohydrates: } \\
24.6-66.78 \mathrm{~g} / 100 \mathrm{~g} \text { (highest } \\
\text { in Macroleptiota procera) }\end{array}$ & [135] \\
\hline $\begin{array}{l}\text { Boletus aereus Bull., Boletus edulis } \\
\text { Bull., Boletus reticulatus Schaeff. }\end{array}$ & Wild mushrooms & $\begin{array}{l}\text { Bragança, } \\
\text { Northeast Portugal }\end{array}$ & $\begin{array}{l}\text { Protein: } \\
\text { 17.86-22.57 g/100 g } \\
\text { (highest in Boletus } \\
\text { reticulatus); carbohydrates: } \\
55.16-72.83 \mathrm{~g} / 100 \mathrm{~g} \\
\text { (highest in Boletus aereus } \\
\text { Bull. }\end{array}$ & [136] \\
\hline Candida valida & $\begin{array}{l}\text { Edible yeast isolated } \\
\text { from babies' weaning } \\
\text { food produced from } \\
\text { fermented corn (Ogi) } \\
\text { and grown on synthetic } \\
\text { medium and cane } \\
\text { molasses }\end{array}$ & Japan & $\begin{array}{l}\text { Protein: } 42.6-44.3 \% \\
\text { (highest when cultured } \\
\text { using cane molasses); } \\
\text { carbohydrate: } 26.9-28.8 \% \\
\text { (highest when cultured } \\
\text { using synthetic medium) }\end{array}$ & [137] \\
\hline Polyporus tenuiculus & $\begin{array}{l}\text { Cultivated mushroom } \\
\text { (cultivated in } \\
\text { supplemented and } \\
\text { nonsupplemented } \\
\text { wheat straw and } \\
\text { willow sawdust) }\end{array}$ & Argentina & $\begin{array}{l}\text { Protein: } 15.1-22.5 \% \\
\text { (highest when cultivated } \\
\text { using wheat straw } \\
\text { supplemented with } \\
\text { soybean flour }(5 \%) \text { and } \\
\text { wheat brand }(15 \%) \text { ); } \\
\text { carbohydrate: } 47.2-51.6 \% \\
\text { (highest when cultivated } \\
\text { using willow sawdust) }\end{array}$ & [138] \\
\hline Terfezia boudieri & Wild desert truffle & $\begin{array}{l}\text { Ben guerdane, } \\
\text { Southeast Tunisia }\end{array}$ & $\begin{array}{l}\text { Protein: } 10.5 \%, 15.4 \% \text { total } \\
\text { sugars }\end{array}$ & [139] \\
\hline Terfezia boudieri & Wild desert truffle & $\begin{array}{l}\text { Hilvan- Sanliurfa, } \\
\text { Yenice/Ceylanpinar/ } \\
\text { Sanliurfa, } \\
\text { Polatlı/Ceylanpinar/ } \\
\text { Sanliurfa, } \\
\text { Kiziltepe-Mardin } \\
\text { and Malatya from } \\
\text { Southeast of Turkey }\end{array}$ & $\begin{array}{l}\text { Protein } 1.40-2.73 \mathrm{~g} / 100 \mathrm{~g} \\
\text { carbohydrate: } \\
4.84-12.30 \mathrm{~g} / 100 \mathrm{~g} \text { (highest } \\
\text { from Kiziltepe/Mardin) }\end{array}$ & {$[140]$} \\
\hline
\end{tabular}


Table 2. Cont

\begin{tabular}{|c|c|c|c|c|}
\hline $\begin{array}{l}\text { Fungal } \\
\text { Species }\end{array}$ & Wild or Cultivated & Location & Composition & References \\
\hline Astraeus hygromatricus & Wild edible fungus & South-west India & $\begin{array}{l}11.71 \% \text { and } 4.66 \% \text { protein } \\
\text { from inner and outer part } \\
\text { of the fruit bodies, } 29.48 \% \\
\text { and } 35.41 \% \text { carbohydrate } \\
\text { from inner and outer fruit } \\
\text { bodies }\end{array}$ & [141] \\
\hline Pleurotus ostreatus & $\begin{array}{l}\text { Cultivated mushroom } \\
\text { (cultivated on oat straw } \\
\text { (control), blank paper } \\
\text { scraps and printed } \\
\text { paper scraps) }\end{array}$ & Portugal & $\begin{array}{l}\text { Protein contents: } \\
9.29-14.7 \mathrm{~g} / 100 \mathrm{~g} \text { (highest } \\
\text { when cultivated on oat } \\
\text { straw; Carbohydrate } \\
\text { contents: } 73.2-78.6 \mathrm{~g} / 100 \mathrm{~g} \\
\text { (highest when cultivated in } \\
\text { printed paper) }\end{array}$ & [142] \\
\hline Pleurotus florida and P. eous & $\begin{array}{l}\text { Cultivated mushrooms } \\
\text { (cultivated on paddy } \\
\text { straw that has been } \\
\text { added with either } \\
\text { chicken manure, rice } \\
\text { bran, wheat bran, } \\
\text { black gram, green gram, } \\
\text { or horse gram.) }\end{array}$ & Tamil-Nadu, India & $\begin{array}{l}\text { Protein contents: } 3.4-35.2 \% \\
\text { dwt. (highest when } \\
\text { cultivated on paddy straw } \\
\text { with chicken manure); } \\
\text { carbohydrate contents: } \\
31-63.8 \% \text { dwt. (highest } \\
\text { when cultivated on paddy } \\
\text { straw with green gram) }\end{array}$ & [143] \\
\hline $\begin{array}{l}\text { Boletus edulis, Boletus mirabilis, } \\
\text { and Lactarius deliciosus }\end{array}$ & Wild mushrooms & $\begin{array}{l}\text { KwaZulu-Natal, } \\
\text { South Africa }\end{array}$ & $\begin{array}{l}\text { Protein contents: } \\
17.5-39.0 \% \text { (highest in } B . \\
\text { edulis); carbohydrate } \\
\text { content: } 51.7-76.0 \% \\
\text { (highest in } \text { L. deliciosus) }\end{array}$ & [144] \\
\hline Pleurotus pulmonarius & Cultivated mushroom & Sao Paolo, Brazil & $\begin{array}{l}\text { Protein contents: } 31 \% \text { in } \\
\text { Basodioma, 32\% in } \\
\text { Mycelium; Carbohydrate } \\
\text { contents: } 30 \% \text { of the } \\
\text { aqueous solution }\end{array}$ & [145] \\
\hline
\end{tabular}

\footnotetext{
Pleurotus eryngii, Dictyophora indusiata (Vent. ex Pers) Fisch, Agrocybe aegerita, Ganoderma lucidum (Leyss. ex Fr.) Karst., Yanshan Agaric, Pholiota nameko Ito ex Imai., Hericium erinaceus, Copyinds comatus (MUII. Fr) gray, Tremella, Cordyceps militaris, Lentinus edodes (Berk.) Sing, Auricularia auricula (L.ex Hook.) under wood, Agaricus blazei Murrill, Volvariella volvacea (Bull.:Fr.) Sing., Morchella esculenta, Griflola frondosa, Arimillaria mellea, Boletus, Russula vinosa Lindblad, and Sparassis crispa.
}

Protein contents: 9.31-37.23\% (highest in Tricholoma Shiitake); Carbohydrate contents: $0.54-37.23 \%$ (highest in Pleurotus eryngii); Ganoderma lucidum (Leyss ex Fr.) Karst. Yashan has the lowest protein and carbohydrate contents.

\section{Antiobesity and Antidiabetic Abilities of Fungi}

Fungi especially edible mushroom, besides its well documented antioxidant capacities, they have also shown boost body immunity. The habit of consistent consumption of edible fungi is effective in the treatment of several medical conditions, such as obesity, and edible fungi could be a good candidate to be applied in future pharmaceutical or 
nutraceutical applications $[147,148]$. Recently, in a study on obese mice, water extracts of Pleurotus citrinopileatus appeared to be effective in reducing the mice weight and helped to improve glucose tolerance and reduce the triglycerides, cholesterol and low-density lipoprotein (LDP) [149]. In an earlier study on obese mice, the fungus Cordyceps militaris played an important cofactor role by fermenting mulberry leaves forming a fungal-plant complex with the ability to rude adipose tissue and decrease LDP [150]. The ascomycete fungi Eurotium cristatum reported having the reduced obesity effect on mice by regulating the mice stomach normal flora [151]. While metabolites extracted from Nigrospora oryzae isolated from plan leaf contain abscisic acid compounds with antidiabetic properties, these fungus extracts show the ability to reduce blood sugar in diabetic [152]. Besides its antioxidant and antiproliferation abilities [91], the tiger milk mushroom showed potential antidiabetic characteristic in its genomes with antiglycation activity medium molecular weight compound with the ability to inhibit lysine in human serum albumin [153]. Peptides with alpha-amylase and alpha glycosidase inhibitory activity identified in the fungus Aspergillus awamori showing the potential of this endophytic fungi with a good biomaterial to be considered for scaled-up production of that peptidase as antidiabetic medication [154]. Recently, Calvatia gigantean revealed promising antidiabetic properties. In an amylase inhibitory test, the fungus revealed the ability to inhibit half of the enzyme (alpha-amylase) at $0.46 \mu \mathrm{g} / \mathrm{mL}$ with 90 times more efficient than the standard acarbose standard drug [155]. group of fungal isolates from medicinal plants belong to Alternaria, Fusarium and Aspergillus sp. showed a promising ability to inhibit $\alpha$-glucosidase enzyme with potential antidiabetic characteristics [156]. Ganoderma lucidium showed promising antidiabetic/antioxidant abilities in comparison to other medicinal mushroom used traditionally in China [157]. Despite all the studies highlighted above and other studies done earlier which showed promising antidiabetic activity for proteins, peptides and polysaccharides from fungal sources, many more studies on the mycotoxicological, clinical level and mode of action are still needed to uncover the safety and efficiency of those molecules.

\section{Fungi as a Biocontrol Agent against Human Pathogen}

Metabolites and their fractions from fungal sources have shown over the years great importance in the discovery of new drugs and compounds with potential antimicrobial properties $[158,159]$. There has been no attempt to discuss the history of antibiotic discovery from fungal sources and the development of antibiotic production in this review, and few earlier reviews have covered this topic [160-163]. Instead, the focus here is to highlight some of the relatively recent promising findings from fungal research with potential antimicrobial characteristics. Aspergillus spp. isolated from river sediment showed antibacterial activities by producing a toxin called gliotoxin that worked actively against pathogenic bacteria like methicillin-resistant Staphylococcus aureus MRSA, Enterococcus faecalis, and Escherichia coli, as well as against human pathogenic yeast such as Candida albicans [164]. Penicillium spp. (P. commune and P. canescen) and Alternaria alternate isolated from olive leaves show the ability to produce phenylethyl alcohol and 3-methyl-1-butanol with both showing the ability to inhibit gram-negative and gram-positive pathogenic bacteria [165]. The mycelial aqueous extract from Ganoderma lucidum demonstrated higher anti-candida activity, the study illustrates the preventive effect of G. lucidum against $C$. albicans and Candida glabrata biofilms [166]. Maitake mushroom (Grifola frondosa) revealed anti-biofilm activity against human pathogenic bacteria Staphylococcus aureus [167]. A 40 KDa unique protein, PEP, that was isolated from edible mushroom Pleurotus eryngii using MALDI-TOF proteomic analysis, was revealed to possess anti-inflammatory properties when tested on LPS-stimulated macrophage in the treatment of colon infection [168]. Another proteomic study, but this time using 1DE LC/MS analysis, showed a group of functional proteins from the edible mushroom Ganoderma lucidum, one of which was the immunomodulatory protein gL18769 with potential function to boost immunity [169]. Oyster mushroom proved to have high antimicrobial characteristics when tested against human pathogenic fungi and bacteria. The mushroom aminophenyl-1thio-3-hydroxypropanoic acid believed to have 
antifungal (against gut yeast Candida albicans, and other pathogenic fungi like Trichosporon cutaneum and Cryptococcus humicola) with antibacterial activities against Staphylococcus aureus and Escherichia coli [170]. The recently promising finding reveals the ability of the synthesized eushearilide which isolated in the first place from the fungus Eupenicillium shearii [171] to inhibit the growth of a wide range of bacteria, including methicillin-resistant Staphylococcus aureus (MRSA) [172]. Recent fungi reported to have anti-biofilm activity are listed in Table 3.

Table 3. Examples of fungal species with anti-biofilm activity.

\begin{tabular}{|c|c|c|}
\hline Fungal Species & Target & Reference \\
\hline $\begin{array}{l}\text { Russula delica, Fistulina hepatica, Mycena rosea, } \\
\text { Leucopaxilus giganteus, and Lepista nuda }\end{array}$ & Pseudomonas aeruginosa & [173] \\
\hline Auricularia auricula & $\begin{array}{l}\text { Pseudomonas aeruginosa and Pseudomonas } \\
\text { fluorescens }\end{array}$ & [174] \\
\hline Lentinus edodes & Streptococcus mutans & [175] \\
\hline Chaetomium globosum & $\begin{array}{c}\text { Staphylococcus aureus, Klebsiella pneumoniae and } \\
\text { Candida albicans }\end{array}$ & [176] \\
\hline Aspergillus nidulans & Candida albicans & [177] \\
\hline Marasmius oreades & $\begin{array}{c}\text { Staphylococcus epidermidis and Pseudomonas } \\
\text { aeruginosa }\end{array}$ & [178] \\
\hline Aspergillus fumigatus & $\begin{array}{c}\text { Staphylococcus aureus, Klebsiella pneumoniae and } \\
\text { Candida albicans }\end{array}$ & [179] \\
\hline Epicoccum nigrum and Alternaria alternata & $\begin{array}{l}\text { Staphylococcus aureus, Pseudomonas aeruginosa, } \\
\text { Escherichia coli and Bacillus subtilis }\end{array}$ & [180] \\
\hline Aspergillus nidulans & Staphylococcus aureus & [181] \\
\hline $\begin{array}{l}\text { Morchella angusticeps, Ganoderma lucidum, Cerioporus } \\
\text { squamosus, Trametes versicolor and Lentinula edodes }\end{array}$ & Enterococcus faecalis & [182] \\
\hline
\end{tabular}

\section{Conclusions and Future Perspective}

Due to the important roles of edible fungi in human and their beneficial applications in medicine and plant protection, further research on SMs from the fungal origin should be intensified to discover and identify novel SMs, understanding their regulatory mechanisms and their physiological function in nature. Only the combination of all factors such as choosing the isolate or the strain, temperature (growth and storage), the medium used to grow the cultivated fungi and the method of choice to extract those compounds can ensure that a certain SM is produced specifically in response to distinct environmental requirements, thus providing a benefit to the fungus. Elucidating the principles behind this complex SM regulatory process using omics will not only allow a deeper understanding of how fungi translate their environmental signals into the biosynthesis of SM, but will also allow for the profiling of novel SMs and a thorough understanding of their potential ecological role. Considering that a vast majority of the known fungi have yet to be cultivated in the laboratory, further efforts in finding the optimum conditions and methods needed to grow such uncultured microorganisms should be pursued. The use of mycosecondary metabolites has made significant improvements in the fields of agriculture, pharmaceutical/drug discovery, medicine, and nutraceuticals industries, especially with the assimilation of modern biotechnology. The numbers of promising applications of fungal metabolites in improving human wellbeing are limitless and continually evolving. There is an urgent demand for the development of new molecular templates for targeted cancer therapeutics and medications to battle multidrug-resistant pathogens. Research must focus on verifying compounds with therapeutic value, and as such, the preclinical and clinical trials on SMs from fungi will lead to faster and more efficient drug development efforts and allow the diversity of these metabolites to be utilized and their application in various 
industries to be considered. Focusing the research community's resources on producing high-quality genome sequences of fungi that yield important and unique SMs and relating these secondary metabolite groups to their annotated biological functions would be a valuable approach. Multiomics high-throughput analyses and information may help us in understanding the production pathways and mode of actions of those vital metabolites.

Author Contributions: Conceptualization, J.R.A.-O.; writing-original draft preparation, J.R.A.-O., N.N.J. and E.I.A.-K. All authors have read and agreed to the published version of the manuscript.

Funding: This work received no external funding.

Institutional Review Board Statement: Not applicable.

Informed Consent Statement: Not applicable.

Data Availability Statement: Not applicable.

Conflicts of Interest: The authors declare no conflict of interest.

\section{References}

1. Keller, N.P. Fungal secondary metabolism: Regulation, function and drug discovery. Nat. Rev. Microbiol. 2019, 17, 167-180. [CrossRef] [PubMed]

2. Demain, A.L. Valuable Secondary Metabolites from Fungi. In Biosynthesis and Molecular genetics of Fungal Secondary Metabolites; Martín, J.-F., garcía-Estrada, C., Zeilinger, S., Eds.; Springer: New York, NY, USA, 2014; pp. 1-15.

3. Calvo, A.M.; Cary, J.W. Association of fungal secondary metabolism and sclerotial biology. Front. Microbiol. 2015, 6. [CrossRef] [PubMed]

4. Kalinina, S.A.; Jagels, A.; Cramer, B.; geisen, R.; Humpf, H.-U. Influence of Environmental Factors on the Production of Penitrems A-F by Penicillium crustosum. Toxins 2017, 9, 210. [CrossRef]

5. Richter, L.; Wanka, F.; Boecker, S.; Storm, D.; Kurt, T.; Vural, Ö.; Süßmuth, R.; Meyer, V. Engineering of Aspergillus niger for the production of secondary metabolites. Fung.. Biol. Biotechnol. 2014, 1, 4. [CrossRef] [PubMed]

6. Bills, G.F.; gloer, J.B. Biologically active secondary metabolites from the fungi. Microbiol. Spectr. 2017, 4, 1087-1119. [CrossRef]

7. Wang, J.B.; St. Leger, R.J.; Wang, C. Chapter Three-Advances in genomics of Entomopathogenic Fungi. Adv. genet. 2016, 94, 67-105. [PubMed]

8. Smith, H.; Doyle, S.; Murphy, R. Filamentous fungi as a source of natural antioxidants. Food Chem. 2015, 185, 389-397. [CrossRef]

9. Zeilinger, S.; gupta, V.K.; Dahms, T.E.S.; Silva, R.N.; Singh, H.B.; Upadhyay, R.S.; gomes, E.V.; Tsui, C.K.-M.; Nayak, S.C. Friends or foes? Emerging insights from fungal interactions with plants. FEMS Microbiol. Rev. 2016, 40, 182-207. [CrossRef]

10. Schmidt-Dannert, C. Biocatalytic portfolio of Basidiomycota. Curr. Opin. Chem. Biol. 2016, 31, 40-49. [CrossRef] [PubMed]

11. Zhang, L. Secondary metabolites and bioactivities from higher fungi in China. Mini. Rev. Med. Chem. 2015, 15, 157-177. [CrossRef]

12. Kalač, P. Chapter 1-Introduction. In Edible Mushrooms; Kalač, P., Ed.; Academic Press: Cambridge, MA, USA, 2016; pp. 1-6.

13. Sabaratnam, V.; Kah-Hui, W.; Naidu, M.; David, P.R. Neuronal Health-Can Culinary and Medicinal Mushrooms Help? J. Tradit. Complem. Med. 2013, 3, 62-68. [CrossRef]

14. Thatoi, H.; Singdevsachan, S.K.; Patra, J.K. Chapter 5-Prebiotics and Their Production From Unconventional Raw Materials (Mushrooms). In Therapeutic, Probiotic, and Unconventional Food; Grumezescu, A.M., Holban, A.M., Eds.; Academic Press: Cambridge, MA, USA, 2018; pp. 79-99.

15. Miles, P.G.; Chang, S.-T. Mushrooms: Cultivation, Utritional Value, Medicinal Effect, and Environmental Impact; CRC Press: Boca Raton, FL, USA, 2004.

16. Larmkie, H.L.; Torgbor, J.P.-N.; Yaa, B.A.M.; Christopher, S.; Matilda, D. Sensory Attributes of Three Edible Tropical Mushrooms and Their Use in Formulating Food Products for Children 2-5 Years Old. Int. J. Nutr. Food. Sci. 2018, 7, 100-109. [CrossRef]

17. Gupta, S.; Summuna, B.; gupta, M.; Annepu, S.K. Edible Mushrooms: Cultivation, Bioactive Molecules, and Health Benefits. In Bioactive Molecules in Food; Mérillon, J.-M., Ramawat, K.G., Eds.; Springer International Publishing: Cham, Switzerland, 2018; pp. 1-33.

18. Al-Obaidi, J.R. Proteomics of edible mushrooms: A mini-review. Electrophoresis 2016, 37, 1257-1263. [CrossRef]

19. Lee, S.; Ryoo, R.; Choi, J.H.; Kim, J.-H.; Kim, S.-H.; Kim, K.H. Trichothecene and tremulane sesquiterpenes from a hallucinogenic mushroom gymnopilus junonius and their cytotoxicity. Arch. Pharm. Res. 2020, 43, 214-223. [CrossRef] [PubMed]

20. Prados-Rosales, R.; Toriola, S.; Nakouzi, A.; Chatterjee, S.; Stark, R.; gerfen, G.; Tumpowsky, P.; Dadachova, E.; Casadevall, A. Structural Characterization of Melanin Pigments from Commercial Preparations of the Edible Mushroom Auricularia auricula. J. Agric. Food Chem. 2015, 63, 7326-7332. [CrossRef]

21. De Souza, R.A.; Kamat, N.M.; Nadkarni, V.S. Purification and characterisation of a sulphur rich melanin from edible mushroom Termitomyces albuminosus Heim. Mycology 2018, 9, 296-306. [CrossRef]

22. Lindequist, U.; Niedermeyer, T.H.; Jülich, W.-D. The pharmacological potential of mushrooms. Evid. Based Complement. Altern. Med. 2005, 2, 285-299. [CrossRef] [PubMed] 
23. Wu, Y.; Choi, M.-H.; Li, J.; Yang, H.; Shin, H.-J. Mushroom cosmetics: The present and future. Cosmetics 2016, 3, 22. [CrossRef]

24. Nakalembe, I.; Kabasa, J.D.; Olila, D. Comparative nutrient composition of selected wild edible mushrooms from two agroecological zones, Uganda. SpringerPlus 2015, 4, 433. [CrossRef] [PubMed]

25. Khan, I. An Investigation of Potential Marketing Strategies for Entry into the Shiitake Mushroom Industry in Utah. Master's Thesis, Utah State University, Logan, UT, USA, 2011.

26. Ma, G.; Yang, W.; Zhao, L.; Pei, F.; Fang, D.; Hu, Q. A critical review on the health promoting effects of mushrooms nutraceuticals. Food. Sci. Hum. Well. 2018, 7, 125-133. [CrossRef]

27. Al-Obaidi, J.R.; Alobaidi, K.H.; Al-Taie, B.S.; Wee, D.H.-S.; Hussain, H.; Jambari, N.N.; Ahmad-Kamil, E.I.; Ariffin, N.S. Uncovering Prospective Role and Applications of Existing and New Nutraceuticals from Bacterial, Fungal, Algal and Cyanobacterial, and Plant Sources. Sustainability 2021, 13, 3671. [CrossRef]

28. Newman, D.J. Predominately Uncultured Microbes as Sources of Bioactive Agents. Front. Microbiol. 2016, 7. [CrossRef]

29. Andersen, M.R.; Nielsen, J.B.; Klitgaard, A.; Petersen, L.M.; Zachariasen, M.; Hansen, T.J.; Blicher, L.H.; gotfredsen, C.H.; Larsen, T.O.; Nielsen, K.F. Accurate prediction of secondary metabolite gene clusters in filamentous fungi. Proc. Natl. Acad. Sci. USA 2013, 110, E99-E107. [CrossRef]

30. Künzler, M. How fungi defend themselves against microbial competitors and animal predators. PLoS Pathog. 2018, 14, 1-10. [CrossRef]

31. Goyal, S.; Ramawat, K.G.; Mérillon, J.-M. Different Shades of Fungal Metabolites: An Overview. In Fungal Metabolites; Mérillon, J.-M., Ramawat, K.G., Eds.; Springer International Publishing: Cham, Switerland, 2017; pp. 1-29.

32. Naranjo-Ortiz, M.A.; gabaldón, T. Fungal evolution: Major ecological adaptations and evolutionary transitions. Biol. Rev. 2019, 94, 1443-1476. [CrossRef]

33. Soldatou, S.; Eldjarn, G.H.; Huerta-Uribe, A.; Rogers, S.; Duncan, K.R. Linking biosynthetic and chemical space to accelerate microbial secondary metabolite discovery. FEMS Microbiol. Lett. 2019, 366. [CrossRef]

34. Beekman, A.M.; Barrow, R.A. Fungal metabolites as pharmaceuticals. Aust. J. Chem. 2014, 67, 827-843. [CrossRef]

35. Wasser, S. Medicinal mushroom science: Current perspectives, advances, evidences, and challenges. Biomed. J. 2014, 37, 345-356. [CrossRef]

36. Qin, D.-W.; Han, C. Medicinal and edible fungi as an alternative medicine for treating age-related disease. Evid. Based Complement. Altern. Med. eCAM 2014, 2014, 638561. [CrossRef]

37. Sánchez, C. Bioactives from Mushroom and Their Application. In Food Bioactives: Extraction and Biotechnology Applications; Puri, M., Ed.; Springer International Publishing: Cham, Switzerland, 2017; pp. $23-57$.

38. Mahmood, R.I.; Abbass, A.K.; Al-Saffar, A.Z.; Al-Obaidi, J.R. An in vitro cytotoxicity of a novel pH-Sensitive lectin loadedcockle shell-derived calcium carbonate nanoparticles against MCF-7 breast tumour cell. J. Drug Deliv. Sci. Technol. 2021, 61, 102230. [CrossRef]

39. Valverde, M.E.; Hernández-Pérez, T.; Paredes-López, O. Edible mushrooms: Improving human health and promoting quality life. Int. J. Microbiol. 2015, 2015, 376387. [CrossRef]

40. Phan, C.-W.; Tan, E.Y.-Y.; Sabaratnam, V. Bioactive Molecules in Edible and Medicinal Mushrooms for Human Wellness. In Bioactive Molecules in Food; Mérillon, J.-M., Ramawat, K.G., Eds.; Springer International Publishing: Cham, Switzerland, 2018; pp. 1-24.

41. Chowdhury, M.M.H.; Kubra, K.; Ahmed, S.R. Screening of antimicrobial, antioxidant properties and bioactive compounds of some edible mushrooms cultivated in Bangladesh. Ann. Clin. Microbiol. Antimicrob. 2015, 14, 8. [CrossRef]

42. Kumaran, R.S.; Jung, H.; Kim, H.J. In vitro screening of taxol, an anticancer drug produced by the fungus, Colletotrichum capsici. Eng. Life Sci. 2011, 11, 264-271. [CrossRef]

43. Gao, P.; Hirano, T.; Chen, Z.; Yasuhara, T.; Nakata, Y.; Sugimoto, A. Isolation and identification of C-19 fatty acids with anti-tumor activity from the spores of ganoderma lucidum (reishi mushroom). Fitoterapia 2012, 83, 490-499. [CrossRef]

44. Frisvad, J.C.; Houbraken, J.; Popma, S.; Samson, R.A. Two new Penicillium species Penicillium buchwaldii and Penicillium spathulatum, producing the anticancer compound asperphenamate. FEMS Microbiol. Lett. 2013, 339, 77-92. [CrossRef]

45. Akanbi, M.H.J.; Post, E.; van Putten, S.M.; de Vries, L.; Smisterova, J.; Meter-Arkema, A.H.; Wösten, H.A.B.; Rink, R.; Scholtmeijer, K. The antitumor activity of hydrophobin SC3, a fungal protein. Appl. Microbiol. Biotechnol. 2013, 97, 4385-4392. [CrossRef]

46. Chakravarthi, B.V.S.K.; Sujay, R.; Kuriakose, G.C.; Karande, A.A.; Jayabaskaran, C. Inhibition of cancer cell proliferation and apoptosis-inducing activity of fungal taxol and its precursor baccatin III purified from endophytic Fusarium solani. Cancer Cell Int. 2013, 13, 105. [CrossRef]

47. Chang, Y.-C.; Hsiao, Y.-M.; Wu, M.-F.; Ou, C.-C.; Lin, Y.-W.; Lue, K.-H.; Ko, J.-L. Interruption of Lung Cancer Cell Migration and Proliferation by Fungal Immunomodulatory Protein FIP-fve from Flammulina velutipes. J. Agric. Food Chem. 2013, 61, 12044-12052. [CrossRef]

48. Ding, X.; Hou, Y.; Zhu, Y.; Wang, P.; Fu, L.; Zhu, H.; Zhang, N.; Qin, H.; Qu, W.; Wang, F.; et al. Structure elucidation, anticancer and antioxidant activities of a novel polysaccharide from gomphus clavatus gray. Oncol. Rep. 2015, 33, 3162-3170. [CrossRef] [PubMed]

49. Xu, H.; Kong, Y.-Y.; Chen, X.; guo, M.-Y.; Bai, X.-H.; Lu, Y.-J.; Li, W.; Zhou, X.-W. Recombinant FIP-gat, a Fungal Immunomodulatory Protein from ganoderma atrum, Induces growth Inhibition and Cell Death in Breast Cancer Cells. J. Agric. Food Chem. 2016, 64, 2690-2698. [CrossRef] 
50. Pushparajah, V.; Fatima, A.; Chong, C.H.; gambule, T.Z.; Chan, C.J.; Ng, S.T.; Tan, C.S.; Fung, S.Y.; Lee, S.S.; Tan, N.H.; et al. Characterisation of a New Fungal Immunomodulatory Protein from Tiger Milk mushroom, Lignosus rhinocerotis. Sci. Rep. 2016, 6, 30010. [CrossRef]

51. Wang, Y.; Compton, C.; Rankin, G.O.; Cutler, S.J.; Rojanasakul, Y.; Tu, Y.; Chen, Y.C. 3-Hydroxyterphenyllin, a natural fungal metabolite, induces apoptosis and S phase arrest in human ovarian carcinoma cells. Int. J. Oncol. 2017, 50, 1392-1402. [CrossRef] [PubMed]

52. Zhou, R.; Han, Y.-J.; Zhang, M.-H.; Zhang, K.-R.; Ng, T.B.; Liu, F. Purification and characterization of a novel ubiquitin-like antitumour protein with hemagglutinating and deoxyribonuclease activities from the edible mushroom Ramaria botrytis. $A M B$ Express 2017, 7, 47. [CrossRef]

53. Xie, Y.; Li, S.; Sun, L.; Liu, S.; Wang, F.; Wen, B.; Sun, L.; Fang, X.; Chai, Y.; Cao, H.; et al. Fungal Immunomodulatory Protein from Nectria haematococca Suppresses growth of Human Lung Adenocarcinoma by Inhibiting the PI3K/Akt Pathway. Int. J. Mol. Sci. 2018, 19, 3429. [CrossRef]

54. Matuszewska, A.; Jaszek, M.; Stefaniuk, D.; Ciszewski, T.; Matuszewski, Ł. Anticancer, antioxidant, and antibacterial activities of low molecular weight bioactive subfractions isolated from cultures of wood degrading fungus Cerrena unicolor. PLoS ONE 2018, 13, e0197044. [CrossRef]

55. Sheeba, H.; Ali, M.S.; Anuradha, V. In-vitro Anti-cancer Activity of Endophytic Fungi Isolated from Ziziphus mauritiana in Cervical Cancer Cell Line. Eur. J. Med. Plants 2020, 31, 38-48. [CrossRef]

56. Li, T.-H.; Hou, C.-C.; Chang, C.L.-T.; Yang, W.-C. Anti-Hyperglycemic Properties of Crude Extract and Triterpenes from Poria cocos. Evid. Based Complement. Altern. Med. eCAM 2011, 2011, 128402. [CrossRef]

57. Huang, H.-Y.; Korivi, M.; Chaing, Y.-Y.; Chien, T.-Y.; Tsai, Y.-C. Pleurotus tuber-regium Polysaccharides Attenuate Hyperglycemia and Oxidative Stress in Experimental Diabetic Rats. Evid. Based Complement. Alternat. Med. 2012, 2012, 856381. [CrossRef] [PubMed]

58. Kang, M.-G.; Yi, S.-H.; Lee, J.-S. Production and Characterization of a New $\alpha$-Glucosidase Inhibitory Peptide from Aspergillus oryzae N159-1. Mycobiology 2013, 41, 149-154. [CrossRef]

59. Stojkovic, D.; Smiljkovic, M.; Ciric, A.; glamoclija, J.; Van griensven, L.; Ferreira, I.C.F.R.; Sokovic, M. An insight into antidiabetic properties of six medicinal and edible mushrooms: Inhibition of $\alpha$-amylase and $\alpha$-glucosidase linked to type-2 diabetes. S. Afr. J. Bot. 2019, 120, 100-103. [CrossRef]

60. Chang, C.-J.; Lin, C.-S.; Lu, C.-C.; Martel, J.; Ko, Y.-F.; Ojcius, D.M.; Tseng, S.-F.; Wu, T.-R.; Chen, Y.-Y.M.; Young, J.D.; et al. Ganoderma lucidum reduces obesity in mice by modulating the composition of the gut microbiota. Nature Commun. 2015, 6, 7489. [CrossRef]

61. Bugeda, A.; garrigues, S.; gandía, M.; Manzanares, P.; Marcos, J.F.; Coca, M. The Antifungal Protein AfpB Induces Regulated Cell Death in Its Parental Fungus Penicillium digitatum. mSphere 2020, 5, e00595-20. [CrossRef] [PubMed]

62. Gashaw, G.; Fassil, A.; Redi, F. Evaluation of the Antibacterial Activity of Pleurotus spp. Cultivated on Different Agricultural Wastes in Chiro, Ethiopia. Int. J. Microbiol. 2020, 2020, 9312489. [CrossRef]

63. Souza Filho, P.F.; Nair, R.B.; Andersson, D.; Lennartsson, P.R.; Taherzadeh, M.J. Vegan-mycoprotein concentrate from peaprocessing industry byproduct using edible filamentous fungi. Fungal Biol. Biotechnol. 2018, 5, 1-10.

64. Rózsa, S.; Măniuțiu, D.-N.; Poșta, G.; gocan, T.-M.; Andreica, I.; Bogdan, I.; Rózsa, M.; Laza, V. Influence of the Culture Substrate on the Agaricus blazei Murrill Mushrooms Vitamins Content. Plants 2019, 8, 316. [CrossRef]

65. Uzma, F.; Mohan, C.D.; Hashem, A.; Konappa, N.M.; Rangappa, S.; Kamath, P.V.; Singh, B.P.; Mudili, V.; gupta, V.K.; Siddaiah, C.N.; et al. Endophytic Fungi-Alternative Sources of Cytotoxic Compounds: A Review. Front. Pharmacol. 2018, 9. [CrossRef]

66. Cardwell, G.; Bornman, J.F.; James, A.P.; Black, L.J. A Review of Mushrooms as a Potential Source of Dietary Vitamin, D. Nutrients 2018, 10, 1498. [CrossRef]

67. Kimatu, B.M.; Zhao, L.; Biao, Y.; Ma, G.; Yang, W.; Pei, F.; Hu, Q. Antioxidant potential of edible mushroom (Agaricus bisporus) protein hydrolysates and their ultrafiltration fractions. Food Chem. 2017, 230, 58-67. [CrossRef] [PubMed]

68. Vamanu, E. Determination of antioxidant and antimicrobial properties of Agaricus bisporus from Romanian markets. Analele Univ. Ovid. Const. Seria Chimie 2012, 23, 47-52.

69. Reis, F.S.; Martins, A.; Barros, L.; Ferreira, I.C.F.R. Antioxidant properties and phenolic profile of the most widely appreciated cultivated mushrooms: A comparative study between in vivo and in vitro samples. Food. Chem.Toxicol. 2012, 50, 1201-1207. [CrossRef]

70. Muna, G.A.; John, M.; Benson, M.; Ogoyi, D. Antioxidant properties of cultivated edible mushroom (Agaricus bisporus) in Kenya. Afr. J. Biotechnol. 2015, 14, 1401-1408.

71. Patinho, I.; Saldaña, E.; Selani, M.M.; de Camargo, A.C.; Merlo, T.C.; Menegali, B.S.; de Souza Silva, A.P.; Contreras-Castillo, C.J. Use of Agaricus bisporus mushroom in beef burgers: Antioxidant, flavor enhancer and fat replacing potential. Food. Prod. Process. Nutr. 2019, 1, 7. [CrossRef]

72. Gasecka, M.; Magdziak, Z.; Siwulski, M.; Mleczek, M. Profile of phenolic and organic acids, antioxidant properties and ergosterol content in cultivated and wild growing species of Agaricus. Eur. Food. Res. Technol. 2018, 244, 259-268. [CrossRef]

73. Hetland, G.; Johnson, E.; Lyberg, T.; Kvalheim, G. The mushroom Agaricus blazei Murill elicits medicinal effects on tumor, infection, allergy, and inflammation through its modulation of innate immunity and amelioration of Th1/Th2 imbalance and inflammation. Adv. Pharmacol. Sci. 2011, 2011,1-10. 
74. Shimizu, T.; Kawai, J.; Ouchi, K.; Kikuchi, H.; Osima, Y.; Hidemi, R. Agarol, an ergosterol derivative from Agaricus blazei, induces caspase-independent apoptosis in human cancer cells. Int. J. Oncol. 2016, 48, 1670-1678. [CrossRef]

75. Matsushita, Y.; Furutani, Y.; Matsuoka, R.; Furukawa, T. Hot water extract of Agaricus blazei Murrill specifically inhibits growth and induces apoptosis in human pancreatic cancer cells. BMC Complement. Altern. Med. 2018, 18, 319. [CrossRef] [PubMed]

76. Ohno, S.; Sumiyoshi, Y.; Hashine, K.; Shirato, A.; Kyo, S.; Inoue, M. Phase I clinical study of the dietary supplement, Agaricus blazei Murill, in cancer patients in remission. Evid. Based. Complement. Alternat. Med. 2011, 2011, 192381. [CrossRef]

77. Taofiq, O.; Rodrigues, F.; Barros, L.; Peralta, R.M.; Barreiro, M.F.; Ferreira, I.C.F.R.; Oliveira, M.B.P.P. Agaricus blazei Murrill from Brazil: An ingredient for nutraceutical and cosmeceutical applications. Food. Funct. 2019, 10, 565-572. [CrossRef]

78. Lin, W.-C.; Deng, J.-S.; Huang, S.-S.; Wu, S.-H.; Chen, C.-C.; Lin, W.-R.; Lin, H.-Y.; Huang, G.-J. Anti-inflammatory activity of Sanghuangporus sanghuang mycelium. Int. J. Mol. Sci 2017, 18, 347. [CrossRef] [PubMed]

79. Cai, C.; Ma, J.; Han, C.; Jin, Y.; Zhao, G.; He, X. Extraction and antioxidant activity of total triterpenoids in the mycelium of a medicinal fungus, Sanghuangporus sanghuang. Sci. Rep. 2019, 9, 7418. [CrossRef]

80. Bakir, T.; Karadeniz, M.; Unal, S. Investigation of antioxidant activities of Pleurotus ostreatus stored at different temperatures. Food Sci. Nutr. 2018, 6, 1040-1044. [CrossRef]

81. Gasecka, M.; Mleczek, M.; Siwulski, M.; Niedzielski, P. Phenolic composition and antioxidant properties of Pleurotus ostreatus and Pleurotus eryngii enriched with selenium and zinc. Eur. Food Res. Technol. 2016, 242, 723-732. [CrossRef]

82. Kumari, M.; Taritla, S.; Sharma, A.; Jayabaskaran, C. Antiproliferative and Antioxidative Bioactive Compounds in Extracts of Marine-Derived Endophytic Fungus Talaromyces purpureogenus. Front. Microbiol. 2018, 9. [CrossRef]

83. Caicedo, N.H.; Davalos, A.F.; Puente, P.A.; Rodríguez, A.Y.; Caicedo, P.A. Antioxidant activity of exo-metabolites produced by Fusarium oxysporum: An endophytic fungus isolated from leaves of Otoba gracilipes. Microbiol. Open 2019, 8, e903. [CrossRef]

84. Pan, F.; Su, T.-J.; Cai, S.-M.; Wu, W. Fungal endophyte-derived Fritillaria unibracteata var. wabuensis: Diversity, antioxidant capacities in vitro and relations to phenolic, flavonoid or saponin compounds. Sci. Rep. 2017, 7, 42008. [CrossRef] [PubMed]

85. Toledo, C.; Barroetaveña, C.; Fernandes, Â.; Barros, L.; Ferreira, I. Chemical and antioxidant properties of wild edible mushrooms from native Nothofagus spp. forest, Argentina. Molecules 2016, 21, 1201. [CrossRef] [PubMed]

86. Raman, J.; Jang, K.-Y.; Oh, Y.-L.; Oh, M.; Im, J.-H.; Lakshmanan, H.; Sabaratnam, V. Cultivation and Nutritional Value of Prominent Pleurotus spp.: An Overview. Mycobiology 2021, 49, 1-14. [CrossRef] [PubMed]

87. González-Palma, I.; Escalona-Buendía, H.B.; Ponce-Alquicira, E.; Téllez-Téllez, M.; Gupta, V.K.; Díaz-Godínez, G.; Soriano-Santos, J. Evaluation of the Antioxidant Activity of Aqueous and Methanol Extracts of Pleurotus ostreatus in Different growth Stages. Front. Microbiol. 2016, 7. [CrossRef] [PubMed]

88. Zhang, B.-B.; Cheung, P.C. Use of stimulatory agents to enhance the production of bioactive exopolysaccharide from Pleurotus tuber-regium by submerged fermentation. J. Agric. Food Chem. 2011, 59, 1210-1216. [CrossRef] [PubMed]

89. Zhang, B.-B.; Chen, L.; Cheung, P.C.K. Proteomic insights into the stimulatory effect of Tween 80 on mycelial growth and exopolysaccharide production of an edible mushroom Pleurotus tuber-regium. Biotechnol. Lett. 2012, 34, 1863-1867. [CrossRef]

90. Yap, H.-Y.Y.; Fung, S.-Y.; Ng, S.-T.; Tan, C.-S.; Tan, N.-H. Shotgun proteomic analysis of tiger milk mushroom (Lignosus rhinocerotis) and the isolation of a cytotoxic fungal serine protease from its sclerotium. J. Ethnopharmacol. 2015, 174, 437-451. [CrossRef]

91. Nallathamby, N.; Phan, C.-W.; Seow, S.L.-S.; Baskaran, A.; Lakshmanan, H.; Abd Malek, S.N.; Sabaratnam, V. A Status Review of the Bioactive Activities of Tiger Milk Mushroom Lignosus rhinocerotis (Cooke) Ryvarden. Front. Pharmacol. 2018, 8. [CrossRef]

92. Chai, Y.; Wang, G.; Fan, L.; Zhao, M. A proteomic analysis of mushroom polysaccharide-treated HepG2 cells. Sci. Rep. 2016, 6, 23565. [CrossRef] [PubMed]

93. Matuszewska, A.; Stefaniuk, D.; Jaszek, M.; Pięt, M.; Zając, A.; Matuszewski, Ł.; Cios, I.; grąz, M.; Paduch, R.; Bancerz, R. Antitumor potential of new low molecular weight antioxidative preparations from the white rot fungus Cerrena unicolor against human colon cancer cells. Sci. Rep. 2019, 9, 1975. [CrossRef] [PubMed]

94. Sezer, Y.Ç.; Süfer, Ö.; Sezer, G. Extraction of phenolic compounds from oven and microwave dried mushrooms (Agaricus bisporus and Pleurotus ostreatus) by using methanol, ethanol and aceton as solvents. Indian J. Pharm. Educ. Res. 2017, 51, 393-397. [CrossRef]

95. Boonsong, S.; Klaypradit, W.; Wilaipun, P. Antioxidant activities of extracts from five edible mushrooms using different extractants. Agric. Nat. Resour. 2016, 50, 89-97. [CrossRef]

96. Gebreyohannes, G.; Nyerere, A.; Bii, C.; Sbhatu, D.B. Investigation of antioxidant and antimicrobial activities of different extracts of auricularia and Termitomyces species of mushrooms. Sci. World J. 2019, 2019, 1-10. [CrossRef]

97. Abdullah, N.; Ismail, S.M.; Aminudin, N.; Shuib, A.S.; Lau, B.F. Evaluation of selected culinary-medicinal mushrooms for antioxidant and ACE inhibitory activities. Evid. Based. Complement. Alternat. Med. 2012, 2012, 1-12. [CrossRef]

98. Zhou, J.; Diao, X.; Wang, T.; Chen, G.; Lin, Q.; Yang, X.; Xu, J. Phylogenetic diversity and antioxidant activities of culturable fungal endophytes associated with the mangrove species Rhizophora stylosa and R. mucronata in the South China Sea. PLoS ONE 2018, 13, 1-18. [CrossRef]

99. Cui, J.-L.; guo, T.-T.; Ren, Z.-X.; Zhang, N.-S.; Wang, M.-L. Diversity and Antioxidant Activity of Culturable Endophytic Fungi from Alpine Plants of Rhodiola crenulata, R. angusta, and R. sachalinensis. PLoS ONE 2015, 10, e0118204. [CrossRef]

100. Wu, F.; Zhou, C.; Zhou, D.; Ou, S.; Zhang, X.; Huang, H. Structure characterization of a novel polysaccharide from Hericium erinaceus fruiting bodies and its immunomodulatory activities. Food Funct. 2018, 9, 294-306. [CrossRef] 
101. Zeng, X.; Ling, H.; Yang, J.; Chen, J.; guo, S. Proteome analysis provides insight into the regulation of bioactive metabolites in Hericium erinaceus. Gene 2018, 666, 108-115. [CrossRef]

102. Vidović, S.S.; Mujić, I.O.; Zeković, Z.P.; Lepojević, Ž.D.; Tumbas, V.T.; Mujić, A.I. Antioxidant properties of selected Boletus mushrooms. Food Biophys. 2010, 5, 49-58. [CrossRef]

103. Yuswan, M.; Al-Obaidi, J.R.; Rahayu, A.; Sahidan, S.; Shazrul, F.; Fauzi, D. New bioactive molecules with potential antioxidant activity from various extracts of wild edible gelam mushroom (Boletus spp.). Adv. Biosci. Biotechnol. 2015, 6, 320. [CrossRef]

104. Jaworska, G.; Pogoń, K.; Skrzypczak, A.; Bernaś, E. Composition and antioxidant properties of wild mushrooms Boletus edulis and Xerocomus badius prepared for consumption. J. Food Sci. Technol. 2015, 52, 7944-7953. [CrossRef]

105. Zhang, L.; Hu, Y.; Duan, X.; Tang, T.; Shen, Y.; Hu, B.; Liu, A.; Chen, H.; Li, C.; Liu, Y. Characterization and antioxidant activities of polysaccharides from thirteen boletus mushrooms. Int. J. Biol. Macromol. 2018, 113, 1-7. [CrossRef]

106. Song, H.-Y.; Kim, D.-H.; Kim, J.-M. Comparative transcriptome analysis of dikaryotic mycelia and mature fruiting bodies in the edible mushroom Lentinula edodes. Sci. Rep. 2018, 8, 8983. [CrossRef]

107. Jamil, N.A.M.; Rashid, N.M.N.; Hamid, M.H.A.; Rahmad, N.; Al-Obaidi, J.R. Comparative nutritional and mycochemical contents, biological activities and LC/MS screening of tuber from new recipe cultivation technique with wild type tuber of tiger's milk mushroom of species Lignosus rhinocerus. World. J. Microbiol. Biotechnol. 2018, 34, 1. [CrossRef]

108. Pinto, E.; Monteiro, C.; Maia, M.; Faria, M.A.; Lopes, V.; Lameiras, C.; Pinheiro, D. Aspergillus Species and Antifungals Susceptibility in Clinical Setting in the North of Portugal: Cryptic Species and Emerging Azoles Resistance in A. fumigatus. Front. Microbiol. 2018, 9. [CrossRef]

109. Hamed, A.A.; Abdel-Aziz, M.S.; El Hady, F.K.A. Antimicrobial and antioxidant activities of different extracts from Aspergillus unguis SPMD-EGY grown on different media. Bull. Nat. Res. Cent. 2018, 42, 29. [CrossRef]

110. Finnigan, T.J.A.; Wall, B.T.; Wilde, P.J.; Stephens, F.B.; Taylor, S.L.; Freedman, M.R. Mycoprotein: The Future of Nutritious Nonmeat Protein, a Symposium Review. Curr. Dev. Nutr. 2019, 3. [CrossRef] [PubMed]

111. Souza Filho, P.F.; Andersson, D.; Ferreira, J.A.; Taherzadeh, M.J. Mycoprotein: Environmental impact and health aspects. World J. Microbiol. Biotechnol. 2019, 35, 147. [CrossRef] [PubMed]

112. Lin, Y.; Zeng, H.; Wang, K.; Lin, H.; Li, P.; Huang, Y.; Zhou, S.; Zhang, W.; Chen, C.; Fan, H. Microwave-assisted aqueous two-phase extraction of diverse polysaccharides from Lentinus edodes: Process optimization, structure characterization and antioxidant activity. Int. J. Biol. Macromol. 2019, 136, 305-315. [CrossRef]

113. Chen, X.; Ji, H.; Xu, X.; Liu, A. Optimization of polysaccharide extraction process from grifola frondosa and its antioxidant and anti-tumor research. J. Food Meas. Charact. 2019, 13, 144-153. [CrossRef]

114. Niu, L.-L.; Wu, Y.-R.; Liu, H.-P.; Wang, Q.; Li, M.-Y.; Jia, Q. Optimization of extraction process, characterization and antioxidant activities of polysaccharide from Leucopaxillus giganteus. J. Food Meas. Charact. 2021, 15, 2842-2853. [CrossRef]

115. González, A.; Cruz, M.; Losoya, C.; Nobre, C.; Loredo, A.; Rodríguez, R.; Contreras, J.; Belmares, R. Edible mushrooms as a novel protein source for functional foods. Food Funct. 2020, 11, 7400-7414.

116. Rahmad, N.; Al-Obaidi, J.R.; Rashid, N.M.N.; Zean, N.B.; Yusoff, M.H.Y.M.; Shaharuddin, N.S.; Jamil, N.A.M.; Saleh, N.M. Comparative proteomic analysis of different developmental stages of the edible mushroom Termitomyces heimii. Biol. Res. 2014, 47, 30. [CrossRef]

117. Wang, M.; gu, B.; Huang, J.; Jiang, S.; Chen, Y.; Yin, Y.; Pan, Y.; Yu, G.; Li, Y.; Wong, B.H.C.; et al. Transcriptome and Proteome Exploration to Provide a Resource for the Study of Agrocybe aegerita. PLoS ONE 2013, 8, e56686. [CrossRef]

118. Lu, T.; Bau, T. De novo assembly and characterization of the transcriptome of a wild edible mushroom Leucocalocybe mongolica and identification of SSR markers. Biotechnol. Biotechnol. Equip. 2017, 31, 1148-1159.

119. Yoo, S.-i.; Lee, H.-Y.; Markkandan, K.; Moon, S.; Ahn, Y.J.; Ji, S.; Ko, J.; Kim, S.-J.; Ryu, H.; Hong, C.P. Comparative transcriptome analysis identified candidate genes involved in mycelium browning in Lentinula edodes. BMC Genom. 2019, 20, 121. [CrossRef]

120. Sato, M.; Miyagi, A.; Yoneyama, S.; gisusi, S.; Tokuji, Y.; Kawai-Yamada, M. CE-MS-based metabolomics reveals the metabolic profile of maitake mushroom (Grifola frondosa) strains with different cultivation characteristics. Biosci. Biotechnol. Biochem. 2017, 81, 2314-2322. [CrossRef]

121. Souza Filho, P.F.; Zamani, A.; Taherzadeh, M.J. Edible protein production by filamentous fungi using starch plant wastewater. Waste Biomass. Valor. 2019, 10, 2487-2496. [CrossRef]

122. Tang, C.; Hoo, P.C.-X.; Tan, L.T.-H.; Pusparajah, P.; Khan, T.M.; Lee, L.-H.; goh, B.-H.; Chan, K.-G. golden needle mushroom: A culinary medicine with evidenced-based biological activities and health promoting properties. Front. Pharmacol. 2016, 7, 474. [CrossRef]

123. Zhang, Y.; Li, H.; Hu, T.; Li, H.; Jin, G.; Zhang, Y. Metabonomic profiling in study hepatoprotective effect of polysaccharides from Flammulina velutipes on carbon tetrachloride-induced acute liver injury rats using gC-MS. Int. J. Biol. Macromol. 2018, 110, 285-293. [CrossRef]

124. Jacinto-Azevedo, B.; Valderrama, N.; Henríquez, K.; Aranda, M.; Aqueveque, P. Nutritional value and biological properties of Chilean wild and commercial edible mushrooms. Food Chem. 2021, 356, 129651. [CrossRef]

125. Punitha, S.C.; Rajasekaran, M. Proximate, elemental and gC-MS study of the edible mushroom Volvariella volvacea (Bull Ex Fr) singer. J. Chem. Pharm. Res. 2015, 7, 511-518.

126. Chittaragi, A.; Naika, R.; Vinayaka, K. Nutritive value of few wild mushrooms from the Western ghats of Shivamogga District, Karnataka, India. Asian J. Pharm. Clin. Res. 2014, 7, 50-53. 
127. Vega, A.; Franco, H. Productividad y calidad de los cuerpos fructíferos de los hongos comestibles Pleurotus pulmonarius RN2 y P. djamor RN81 y RN82 cultivados sobre sustratos lignocelulósicos. Inf. Tecnol. 2013, 24, 69-78. [CrossRef]

128. Singdevsachan, S.K.; Patra, J.K.; Thatoi, H. Nutritional and bioactive potential of two wild edible mushrooms (Lentinus sajor-caju and Lentinus torulosus) from Similipal Biosphere Reserve, India. Food Sci. Biotechnol. 2013, 22, 137-145. [CrossRef]

129. Leal, A.R.; Barros, L.; Barreira, J.C.M.; Sousa, M.J.; Martins, A.; Santos-Buelga, C.; Ferreira, I.C.F.R. Portuguese wild mushrooms at the "pharma-nutrition" interface: Nutritional characterization and antioxidant properties. Food Res. Int. 2013, 50, 1-9. [CrossRef]

130. Reis, F.S.; Stojković, D.; Soković, M.; glamočlija, J.; Ćirić, A.; Barros, L.; Ferreira, I.C.F.R. Chemical characterization of Agaricus bohusii, antioxidant potential and antifungal preserving properties when incorporated in cream cheese. Food Res. Int. 2012, 48, 620-626. [CrossRef]

131. Palazzolo, E.; Letizia gargano, M.; Venturella, G. The nutritional composition of selected wild edible mushrooms from Sicily (southern Italy). Int. J. Food Sci. Nutr. 2012, 63, 79-83. [CrossRef]

132. Kumari, D.; Reddy, M.; Upadhyay, R. Nutritional composition and antioxidant activities of 18 different wild Cantharellus mushrooms of Northwestern Himalayas. Food Sci. Technol. Int. 2011, 17, 557-567. [CrossRef]

133. Vaz, J.A.; Barros, L.; Martins, A.; Santos-Buelga, C.; Vasconcelos, M.H.; Ferreira, I.C.F.R. Chemical composition of wild edible mushrooms and antioxidant properties of their water soluble polysaccharidic and ethanolic fractions. Food Chem. 2011, 126, 610-616. [CrossRef]

134. Michael, H.W.; Bultosa, G.; Pant, L.M. Nutritional contents of three edible oyster mushrooms grown on two substrates at Haramaya, Ethiopia, and sensory properties of boiled mushroom and mushroom sauce. Int. J. Food Sci. Technol. 2011, 46, 732-738. [CrossRef]

135. Beluhan, S.; Ranogajec, A. Chemical composition and non-volatile components of Croatian wild edible mushrooms. Food Chem. 2011, 124, 1076-1082. [CrossRef]

136. Heleno, S.A.; Barros, L.; Sousa, M.J.; Martins, A.; Santos-Buelga, C.; Ferreira, I.C.F.R. Targeted metabolites analysis in wild Boletus species. LWT Food Sci. Technol. 2011, 44, 1343-1348. [CrossRef]

137. Kuforiji, O.; Aboaba, O. Application of Candida valida as a protein supplement. J. Food Safety 2010, 30, 969-981. [CrossRef]

138. Omarini, A.; Henning, C.; Ringuelet, J.; Zygadlo, J.A.; Albertó, E. Volatile composition and nutritional quality of the edible mushroom Polyporus tenuiculus grown on different agro-industrial waste. Int. J. Food Sci. Technol. 2010, 45, 1603-1609. [CrossRef]

139. Slama, A.; Neffati, M.; Boudabous, A. Biochemical composition of desert truffle Terfezia boudieri Chatin. In Proceedings of the International Symposium on Medicinal and Aromatic Plants-SIPAM2009 853, Djerba, Tunisia, 26-28 March 2009 ; pp. $285-290$.

140. Dundar, A.; Yesil, O.F.; Acay, H.; Okumus, V.; Ozdemir, S.; Yildiz, A. Antioxidant properties, chemical composition and nutritional value of Terfezia boudieri (Chatin) from Turkey. Food Sci. Technol. Int. 2012, 18, 317-328. [CrossRef]

141. Pavithra, M.; Sridhar, K.R.; greeshma, A.A.; Tomita-Yokotani, K. Bioactive potential of the wild mushroom Astraeus hygrometricus in South-west India. Mycology 2016, 7, 191-202. [CrossRef] [PubMed]

142. Fernandes, Â.; Barros, L.; Martins, A.; Herbert, P.; Ferreira, I.C.F.R. Nutritional characterisation of Pleurotus ostreatus (Jacq. ex Fr.) P. Kumm. produced using paper scraps as substrate. Food Chem. 2015, 169, 396-400. [CrossRef]

143. Sivagurunathan, P.; Sivasankari, S. Influence of Chicken Manure on Biological Efficiency of Pleurotus spp. Waste Biomass Valor. 2015, 6, 23-28. [CrossRef]

144. Rasalanavho, M.; Moodley, R.; Jonnalagadda, S.B. Elemental bioaccumulation and nutritional value of five species of wild growing mushrooms from South Africa. Food Chem. 2020, 319, 126596. [CrossRef] [PubMed]

145. Contato, A.G.; Inácio, F.D.; de Araújo, C.A.V.; Brugnari, T.; Maciel, G.M.; Haminiuk, C.W.I.; Bracht, A.; Peralta, R.M.; de Souza, C.G.M. Comparison between the aqueous extracts of mycelium and basidioma of the edible mushroom Pleurotus pulmonarius: Chemical composition and antioxidant analysis. J. Food Meas. Charact. 2020, 14, 830-837. [CrossRef]

146. Yu, Q.; guo, M.; Zhang, B.; Wu, H.; Zhang, Y.; Zhang, L. Analysis of Nutritional Composition in 23 Kinds of Edible Fungi. J. Food Qual. 2020, 2020, 1-9. [CrossRef]

147. Ganesan, K.; Xu, B. Anti-obesity effects of medicinal and edible mushrooms. Molecules 2018, 23, 2880.

148. Helal, N.A.; Eassa, H.A.; Amer, A.M.; Eltokhy, M.A.; Edafiogho, I.; Nounou, M.I. Nutraceuticals' Novel Formulations: The good, the Bad, the Unknown and Patents Involved. Recent Pat. Drug. Deliv. Formul. 2019, 13, 105-156. [CrossRef]

149. Sheng, Y.; Zhao, C.; Zheng, S.; Mei, X.; Huang, K.; Wang, G.; He, X. Anti-obesity and hypolipidemic effect of water extract from Pleurotus citrinopileatus in C57BL/6J mice. Food Sci. Nutr. 2019, 7, 1295-1301. [CrossRef]

150. Lee, M.R.; Kim, J.E.; Choi, J.Y.; Park, J.J.; Kim, H.R.; Song, B.R.; Choi, Y.W.; Kim, K.M.; Song, H.; Hwang, D.Y. Anti-obesity effect in high-fat-diet-induced obese C57BL/6 mice: Study of a novel extract from mulberry (Morus alba) leaves fermented with Cordyceps militaris. Exp. Ther. Med. 2019, 17, 2185-2193. [CrossRef]

151. Kang, D.; Su, M.; Duan, Y.; Huang, Y. Eurotium cristatum, a potential probiotic fungus from Fuzhuan brick tea, alleviated obesity in mice by modulating gut microbiota. Food Funct. 2019, 10, 5032-5045. [CrossRef]

152. Uzor, P.F.; Osadebe, P.O.; Nwodo, N.J. Antidiabetic activity of extract and compounds from an endophytic fungus Nigrospora oryzae. Pharm. Res. 2017, 67, 308-311. [CrossRef]

153. Yap, H.-Y.Y.; Tan, N.-H.; Ng, S.-T.; Tan, C.-S.; Fung, S.-Y. Inhibition of protein glycation by tiger milk mushroom [Lignosus rhinocerus (Cooke) Ryvarden] and search for potential anti-diabetic activity-related metabolic pathways by genomic and transcriptomic data mining. Front. Pharmacol. 2018, 9, 103. [CrossRef] 
154. Singh, B.; Kaur, A. Antidiabetic potential of a peptide isolated from an endophytic Aspergillus awamori. J. Appl. Microbiol. 2016, 120, 301-311. [CrossRef]

155. Ogbole, O.O.; Nkumah, A.O.; Linus, A.U.; Falade, M.O. Molecular identification, in vivo and in vitro activities of Calvatia gigantea (macro-fungus) as an antidiabetic agent. Mycology 2019, 10, 166-173. [CrossRef]

156. Kaur, A. Evaluation of antidiabetic and antioxidant potential of endophytic fungi isolated from medicinal plants. Int. J. green. Pharm. 2018, 12, 6-14.

157. $\mathrm{Wu}, \mathrm{T} . ; \mathrm{Xu}, \mathrm{B}$. Antidiabetic and antioxidant activities of eight medicinal mushroom species from China. Int. J. Med. Mushrooms. 2015, 17, 129-140. [CrossRef]

158. Zutz, C.; Bandian, D.; Neumayer, B.; Speringer, F.; gorfer, M.; Wagner, M.; Strauss, J.; Rychli, K. Fungi treated with small chemicals exhibit increased antimicrobial activity against facultative bacterial and yeast pathogens. Biomed Res. Int. 2014, 2014, 1-13. [CrossRef]

159. Wiese, J.; Imhoff, J.F. Marine bacteria and fungi as promising source for new antibiotics. Drug Develop. Res. 2019, 80, 24-27. [CrossRef]

160. Aly, A.H.; Debbab, A.; Proksch, P. Fifty years of drug discovery from fungi. Fungal Divers 2011, 50, 3. [CrossRef]

161. Dias, D.A.; Urban, S.; Roessner, U. A historical overview of natural products in drug discovery. Metabolites $2012,2,303-336$.

162. Deshmukh, S.K.; Verekar, S.A.; Bhave, S.V. Endophytic fungi: A reservoir of antibacterials. Front. Microbiol. 2015, 5. [CrossRef]

163. Xu, L.; Meng, W.; Cao, C.; Wang, J.; Shan, W.; Wang, Q. Antibacterial and antifungal compounds from marine fungi. Mar. Drugs 2015, 13, 3479-3513. [CrossRef]

164. Svahn, K.S.; göransson, U.; El-Seedi, H.; Bohlin, L.; Larsson, D.J.; Olsen, B.; Chryssanthou, E. Antimicrobial activity of filamentous fungi isolated from highly antibiotic-contaminated river sediment. Infec. Ecol. Epidemiol. 2012, 2, 11591. [CrossRef]

165. Malhadas, C.; Malheiro, R.; Pereira, J.A.; de Pinho, P.G.; Baptista, P. Antimicrobial activity of endophytic fungi from olive tree leaves. World J. Microbiol. Biotechnol. 2017, 33, 46. [CrossRef]

166. Bhardwaj, A.; gupta, P.; Kumar, N.; Mishra, J.; Kumar, A.; Rakhee; Misra, K. Lingzhi or Reishi Medicinal Mushroom, ganoderma lucidum (Agaricomycetes), Inhibits Candida Biofilms: A Metabolomic Approach. Int. J. Med. Mush. 2017, 19, 685-696. [CrossRef]

167. Gargano, M.L.; Zervakis, G.I.; Isikhuemhen, O.S.; Venturella, G.; Calvo, R.; giammanco, A.; Fasciana, T.; Ferraro, V. Ecology, Phylogeny, and Potential Nutritional and Medicinal Value of a Rare White "Maitake" Collected in a Mediterranean Forest. Diversity 2020, 12, 230. [CrossRef]

168. Yuan, B.; Zhao, L.; Rakariyatham, K.; Han, Y.; gao, Z.; Kimatu, B.M.; Hu, Q.; Xiao, H. Isolation of a novel bioactive protein from an edible mushroom Pleurotus eryngii and its anti-inflammatory potential. Food Funct. 2017, 8, 2175-2183. [CrossRef]

169. Yu, G.-J.; Yin, Y.-L.; Yu, W.-H.; Liu, W.; Jin, Y.-X.; Shrestha, A.; Yang, Q.; Ye, X.-D.; Sun, H. Proteome Exploration to Provide a Resource for the Investigation of ganoderma lucidum. PLoS ONE 2015, 10, e0119439. [CrossRef]

170. Younis, A.M.; Wu, F.-S.; El Shikh, H.H. Antimicrobial activity of extracts of the oyster culinary medicinal mushroom Pleurotus ostreatus (higher basidiomycetes) and identification of a new antimicrobial compound. Int. J. Med. Mushrooms 2015, 17, 579-590. [CrossRef]

171. Hosoe, T.; Fukushima, K.; Takizawa, K.; Itabashi, T.; Kawahara, N.; Vidotto, V.; Kawai, K.-I. A New Antifungal Macrolide, Eushearilide, Isolated from Eupenicillium shearii. J. Antibiot. 2006, 59, 597-600. [CrossRef]

172. Tonoi, T.; Inohana, T.; Sato, T.; Noda, Y.; Ikeda, M.; Akutsu, M.; Murata, T.; Maekawa, Y.; Tanaka, A.; Seki, R. Total Synthesis and Antimicrobial Evaluation of 23-Demethyleushearilide and Extensive Antimicrobial Evaluation of All Synthetic Stereoisomers of (16Z, 20E)-Eushearilide and (16E, 20E)-Eushearilide. Molecules 2019, 24, 3437. [CrossRef]

173. Alves, M.J.; Ferreira, I.C.F.R.; Lourenço, I.; Costa, E.; Martins, A.; Pintado, M. Wild Mushroom Extracts as Inhibitors of Bacterial Biofilm Formation. Pathogens 2014, 3, 667-679. [CrossRef]

174. Bin, L.; Wei, L.; Xiaohong, C.; Mei, J.; Mingsheng, D. In vitro antibiofilm activity of the melanin from Auricularia auricula, an edible jelly mushroom. Ann. Microbiol. 2012, 62, 1523-1530. [CrossRef]

175. Papetti, A.; Signoretto, C.; Spratt, D.A.; Pratten, J.; Lingström, P.; Zaura, E.; Ofek, I.; Wilson, M.; Pruzzo, C.; Gazzani, G. Components in Lentinus edodes mushroom with anti-biofilm activity directed against bacteria involved in caries and gingivitis. Food Funct. 2018, 9, 3489-3499. [CrossRef]

176. Kaur, N.; Arora, D.S. Prospecting the antimicrobial and antibiofilm potential of Chaetomium globosum an endophytic fungus from Moringa oleifera. AMB Express 2020, 10, 206. [CrossRef] [PubMed]

177. Meenambiga, S.; Rajagopal, K. Antibiofilm activity and molecular docking studies of bioactive secondary metabolites from endophytic fungus Aspergillus nidulans on oral Candida albicans. J. Appl. Pharm. Sci. 2018, 8, 37-45.

178. Shomali, N.; Onar, O.; Karaca, B.; Demirtas, N.; Cihan, A.C.; Akata, I.; Yildirim, O. Antioxidant, anticancer, antimicrobial, and antibiofilm properties of the culinary-medicinal fairy ring mushroom, Marasmius oreades (Agaricomycetes). Int. J. Med. Mushrooms 2019, 21, 571-582. [CrossRef]

179. Kaur, N.; Arora, D.S.; Kalia, N.; Kaur, M. Antibiofilm, antiproliferative, antioxidant and antimutagenic activities of an endophytic fungus Aspergillus fumigatus from Moringa oleifera. Mol. Biol. Rep. 2020, 47, 2901-2911. [CrossRef]

180. Qader, M.M.; Hamed, A.A.; Soldatou, S.; Abdelraof, M.; Elawady, M.E.; Hassane, A.S.I.; Belbahri, L.; Ebel, R.; Rateb, M.E. Antimicrobial and Antibiofilm Activities of the Fungal Metabolites Isolated from the Marine Endophytes Epicoccum nigrum M13 and Alternaria alternata 13A. Mar. Drugs 2021, 19, 232. [CrossRef] 
181. Kumar, S.; Kumar, A.; Kaushal, M.; Kumar, P.; Mukhopadhyay, K.; Kumar, A. Fungal-derived xenobiotic exhibits antibacterial and antibiofilm activity against Staphylococcus aureus. Drug Discov. Ther. 2018, 12, 214-223. [CrossRef]

182. Karaca, B.; Cihan, A.Ç.; Akata, I.; Altuner, E.M. Anti-Biofilm and Antimicrobial Activities of Five Edible and Medicinal Macrofungi Samples on Some Biofilm Producing Multi Drug Resistant Enterococcus Strains. Turkish J. Agric. Food Sci. Technol. 2020, 8 , 69-80. [CrossRef] 